\title{
Targeting mitochondrial double-stranded RNAs ameliorates autoimmune characteristics of Sjögren's syndrome
}

Authors: Jimin Yoon ${ }^{1 \dagger}$, Min-Seok Lee ${ }^{1 \dagger}$, Ahsan Ausaf Ali ${ }^{1}$, Ye Rim Oh ${ }^{2}$, Yong Seok Choi ${ }^{2}$, Sujin Kim $^{1}$, Namseok Lee ${ }^{1}$, Se Gwang Jang ${ }^{3}$, Naseem Ahamad ${ }^{4}$, Seung-Ki Kwok ${ }^{5}$, Joon Young Hyon ${ }^{6}$, Seunghee $\mathrm{Cha}^{4}$, Yun Jong Lee ${ }^{7,8^{*}}$, Sung Gap $\mathrm{Im}^{1,9 *}$, Yoosik Kim ${ }^{1,10^{*}}$

\begin{abstract}
Affiliations:
${ }^{1}$ Department of Chemical and Biomolecular Engineering, Korea Advanced Institute of Science and Technology (KAIST), Daejeon 34141, Korea.

${ }^{2}$ Medical Science Research Institute, Seoul National University Bundang Hospital, Seongnam 13620 , Korea.

${ }^{3}$ The Rheumatism Research Center, Catholic Research Institute of Medical Science, College of Medicine, The Catholic University of Korea, Seoul 06591, Korea.

${ }^{4}$ Department of Oral and Maxillofacial Diagnostic Sciences, University of Florida College of Dentistry, Gainesville, FL 32610, USA.

${ }^{5}$ Division of Rheumatology, Department of Internal Medicine, Seoul St. Mary's Hospital, College of Medicine, The Catholic University of Korea, Seoul 06591, Korea.

${ }^{6}$ Department of Ophthalmology, Seoul National University Bundang Hospital, Seongnam 13620, Korea.

${ }^{7}$ Division of Rheumatology, Department of Internal Medicine, Seoul National University Bundang Hospital, Seongnam 13620, Korea.

${ }^{8}$ Department of Internal Medicine, Department of Internal Medicine, Seoul National University College of Medicine, Seoul 03080, Korea.

${ }^{9}$ KI for NanoCentury (KINC), KAIST, Daejeon 34141, Korea.

${ }^{10} \mathrm{KI}$ for Health Science and Technology (KIHST), KAIST, Daejeon 34141, Korea.

$\dagger:$ These authors contributed equally to this work as co-first authors.

*Correspondence should be addressed to Yoosik Kim: Tel: +82-42-350-7312; Fax: +82-42-3503910; Email: ysyoosik@ kaist.ac.kr. Correspondence may also be addressed to Yun Jong Lee: Tel: +82-31-787-7049; Fax: +82-31-787-4051; Email: yn35@snu.ac.kr and Sung Gap Im: Tel: +8242-350-3976; Email: sgim@ kaist.ac.kr.
\end{abstract}

Summary: Mitochondrial double-stranded RNA levels are elevated in the tear and saliva of SS patients. These RNAs promote type I interferon signature, as well as other autoimmune phenotypes in SS. Downregulation of mitochondrial dsRNAs alleviates autoimmune signatures of SS in salivary gland epithelial cells. 


\begin{abstract}
:
Sjögren's syndrome (SS) is a systemic autoimmune disease that targets the exocrine glands, resulting in impaired saliva and tear secretion. To date, type I interferons (IFNs) are increasingly recognized as pivotal mediators in SS, but their endogenous drivers have not been elucidated. Here, we investigate the role of mitochondrial double-stranded RNAs (mt-dsRNAs) in regulating type I IFN response in SS. We find that mt-dsRNAs are elevated in the saliva and tear of SS patients and in salivary glands of non-obese diabetic mice with salivary dysfunction. Using the in-housedeveloped 3D culture, we show that dsRNA stimulation increases mt-dsRNAs expression via JAK1/STAT pathway and facilitates their cytosolic export, which is accompanied by autoimmune signatures observed in SS. We further show that muscarinic receptor ligand acetylcholine or antioxidant resveratrol ameliorates autoimmune characteristics by preventing mt-dsRNAmediated immune activation. In addition, direct suppression of mt-dsRNAs reverses the autoimmune signatures of SS. Altogether, our study underscores the significance of mt-dsRNA upregulation and suggests mt-dsRNAs as an important key to the puzzle of SS.
\end{abstract}




\section{INTRODUCTION}

Sjögren's syndrome (SS) is a slowly progressive autoimmune disorder characterized by lymphocytic infiltration of the exocrine glands, B cell hyperactivity with autoantibodies, and significant loss of secretory function, resulting in oral and eye dryness (Jin et al, 2013; Kroese et al, 2014; Qin et al, 2015). As with most autoimmune disorders, the etiology of SS is not yet fully understood. It is particularly challenging to establish the diagnosis due to individual patients with overlapping disease entities (Stefanski et al, 2017). Moreover, a therapeutic target of SS is unavailable as the treatment options depend on individual disease activity, and SS is diagnosed after the disease has progressed enough to cause significant pain. This delay is problematic because the patients' quality of life is significantly reduced by diverse manifestations of SS as well as numerous complications, including cardiovascular diseases and lymphoma (Stefanski et al., 2017). Hence, it is important to expand our knowledge about the immunopathological mechanisms involved in SS to identify the targets for potential therapies and diagnosis.

One of the most notable features of SS is the type I interferon (IFN) signature (Crow et al, 2019). The type I IFNs can be produced in response to stimulation of pattern recognition receptors (PRRs) such as Toll-Like Receptors (TLRs), Protein Kinase R (PKR), and Melanoma Differentiation-Associated Gene 5 (MDA5) that recognize double-stranded RNAs (dsRNAs) (Huijser \& Versnel, 2021; Kiripolsky \& Kramer, 2018). Indeed, the upregulation of TLRs is often accompanied by increased levels of IFN-stimulated genes (ISGs) and proinflammatory cytokines, including Tumor Necrosis Factor- $\alpha(\mathrm{TNF}-\alpha)$, various interleukins (ILs), and IFN- $\gamma$ in SS patients (Bikker et al, 2010). In particular, salivary glands with extensive mononuclear cell infiltration showed increased expression of type I IFNs and ISGs that are downstream targets of nucleic acidsensing PRRs (Greenwell-Wild et al, 2011; Min et al, 2019). Consequently, the dysregulated 
detection of nucleic acids has been established as one of the autoimmune signatures (Huijser \& Versnel, 2021). Taken together, dsRNA-sensing PRRs may play an important role in engendering autoimmune phenotypes, and investigating both the identity and the roles of PRR-activating dsRNAs in the pathogenesis of SS is critical to understand type I IFN signature in SS.

Historically, long dsRNAs were associated with viral infection (Guo et al, 2019). However, recent evidence has shown that dsRNAs are also expressed endogenously and that they can initiate the immune response by activating PRRs (Ahmad et al, 2018; Kim et al, 2018). Among several types of endogenous dsRNAs, mt-dsRNAs are receiving increasing attention due to their strong biomedical implications in diseases such as alcohol-associated liver disease (Lee et al, 2020b), Huntington's disease (Lee et al, 2020a), and osteoarthritis (Kim et al, 2020). Mitochondria are able to generate dsRNAs due to bidirectional transcription of the mitochondrial genome. When present in the cytosol due to mutations in regulatory proteins or under stress, mt-dsRNAs are recognized by PRRs, such as PKR and MDA5, and initiate the downstream immune response (Dhir et al, 2018; Kim et al., 2018). However, mt-dsRNA regulation and its pathophysiological role in SS still remain uninvestigated. Considering the excess level of oxidative stress and implications of mitochondrial dysfunction in chronic inflammation and tissue damages in SS (Cole et al, 2016; Hu et al, 2009; Pagano et al, 2013; Ryo et al, 2006), we hypothesized that mt-dsRNAs might participate in the activation of PRRs and drive the type I IFN response in SS.

In this study, we investigated the role of mt-dsRNAs in the pathogenesis of SS. We first analyzed the expression of both heavy and light strands of mitochondrial transcripts in saliva and tear samples of SS patients and analyzed the in vivo relevance using non-obese diabetic (NOD) mice with salivary dysfunction. We further investigated the role of mt-dsRNAs on SS-like autoimmune signatures, such as ISG induction, PKR activation, and altered expression of tight 
junction complex (TJC) proteins and Aquaporin-5 (AQP5) water channel protein using salivary gland epithelial cells (SGECs) grown as three-dimensional (3D) spheroids. With the established 3D culture system, we examined whether muscarinic receptor (M3R) ligand acetylcholine (Ach) and antioxidant resveratrol (RES), two small molecules known to improve salivary function, suppressed SS-like autoimmune signatures via mt-dsRNA regulation. Lastly, we analyzed the effect of direct downregulation of mt-dsRNA expression on autoimmune signatures using an inhibitor of mitochondrial RNA polymerase (POLRMT). Together, our study uncovers the key regulatory function of mt-dsRNAs in exacerbating autoimmune characteristics in SS.

\section{RESULTS}

mt-dsRNA levels are elevated in saliva and tears of SS patients and salivary glands of aged NOD mice

We began our investigation by analyzing the expression levels of mt-dsRNAs because increased oxidative stress was reported in both mouse models and human samples of SS (Cole $e t$ al., 2016; Hu et al., 2009; Pagano et al., 2013; Ryo et al., 2006). We first measured mt-dsRNA levels in the saliva and tear samples collected from 73 and 16 study subjects, respectively. We extracted total RNAs from $200 \mu \mathrm{L}$ of saliva and performed strand-specific reverse transcription to analyze the expression of heavy and light strand RNAs separately (Fig 1A). Among 16 RNAs analyzed, 15 of them showed higher median expression levels in all SS patients compared to nonSS sicca patients (Fig 1B). When compared to the non-sicca controls, 13 out of 16 RNAs showed higher expression levels in SS patients. Notably, all examined RNA expression patterns of the nonsicca control were indistinguishable from those of the non-SS sicca group. ANOVA analysis revealed statistically different $(\mathrm{P}$-value $<0.05)$ expressions for 15 out of 16 RNAs. We also 
examined mt-dsRNA expression patterns in 16 tear samples - 8 SS and 8 non-SS sicca subjects (Fig 1C). Similar to the saliva samples, higher levels of mt-dsRNAs were detected in SS. Most RNAs (14/15) tested showed higher expression in SS patients compared to the non-SS sicca controls. Collectively, our results suggest that mt-dsRNA expression patterns can be used to distinguish between non-SS sicca and SS patients.

We further analyzed our data and ranked the five most significant RNAs in tear and saliva samples respectively (Fig S1A and B). From both samples, $C Y T B$ heavy, ND1 light, and CO1 light strands were highly expressed in SS compared to non-SS sicca patients. When we analyzed the association between RNA levels in body fluid and exocrine dysfunction, we found that tear $C Y T B$ light strand levels were negatively correlated to tear production and positively correlated to the corneal and conjunctival damage (Fig S1C-E). Furthermore, salivary ND5 heavy strand transcript levels showed a negative correlation to the stimulated whole salivary flow rates and a positive correlation to lymphocyte infiltration grade (Fig S1F-I).

Next, we investigated the expression of mt-dsRNAs in the epithelial cells of the salivary glands obtained from the NOD mice. Aged NOD mice develop SS-like autoimmune exocrinopathy, making them one of the most powerful tools in deciphering pathologic mechanisms of SS (Park et $a l, 2015)$. In our study, the salivary glands of NOD mice at different ages were analyzed. Since a significant decrease in the salivary flow rate of NOD mice after 17 weeks (Jonsson et al, 2006), we focused our analysis on NOD mice between 16 weeks and 28 weeks of age with 8 weeks mice as controls. We extracted total RNAs from $20 \mu \mathrm{m}$ tissue sections of NOD mice salivary gland and measured the expression of five mt-dsRNAs - Nd1, Nd5, Nd6, Col, and Cytb-which showed the most significant enrichment from the saliva samples of SS patients. Compared to that of 8-weekold NOD mice, expression levels for all mt-dsRNAs except $C y t b$ were higher in NOD mice older 
than 20 weeks (Fig 1D). Of note, due to high variance, ANOVA analysis did not yield any significant change despite a clear increase in the mean expression of the RNAs. Alternatively, we analyzed mt-dsRNA patterns by using single-molecule fluorescent RNA in situ hybridization (RNA-FISH). We clearly found that the expression of both $N d 5$ heavy $(N d 5 H)$ and light strand $(N d 5 L)$ RNAs were elevated in acinar regions of NOD mice submandibular salivary gland tissues aged from 20 weeks to 28 weeks (Fig 1E), compared to the expression in 16-week mice. The increased expression of both heavy and light strand RNAs indicated the induction of mt-dsRNAs. Of note, the negative control probe yielded no signal (Fig 1F).

\section{Antiviral signaling increases mt-dsRNA expression}

Next, we investigated whether stimulating the antiviral signaling and type I IFN pathway in SGECs can elicit mt-dsRNA induction. In order to induce SS-like condition, we transfected SV40 transformed salivary gland-squamous acinar cells (NS-SV-AC) with polyinosinic-polycytidylic acid (poly I:C), a synthetic analog of viral dsRNAs, for PRR stimulation. Repeated administration of poly I:C was reported to elicit IL-7 production and early inflammatory responses in the salivary gland and accelerate the development of SS-like sialadenitis (Deshmukh et al, 2009; Jin et al., 2013). Moreover, poly I:C also led to other key characteristics of SS, such as leukocytic infiltration, antinuclear antibody production, and impaired tear secretion in mice (Zhou et al, 2015). We first analyzed the expression of ISGs associated with SS and the degree of PKR phosphorylation because the expression of PKR was increased in monocytes from IFN-positive SS patients (Maria et al, 2017) and various ISGs, such as MX1, IRF7, and IFI44 showed increased expression in the salivary tissues of the SS model (Kiripolsky \& Kramer, 2018). Our data revealed strong ISGs 
induction, increased PKR phosphorylation, and a consequential decrease in cell viability (Fig S2AC).

We then measured the expression levels of mt-dsRNAs upon poly I:C stimulation. To our surprise, we found that the dsRNA stress to the salivary cells resulted in increased mt-dsRNA expression, both heavy and their complementary light strand RNAs (Fig S2D and E). Direct visualization of ND5 mt-dsRNA via RNA-FISH further confirmed that both heavy and light strand RNAs showed increased expression (Fig S2F). We also analyzed the cytosolic presence of mtdsRNAs to determine if mt-dsRNAs can interact with PRRs such as PKR to augment the antiviral signaling. We isolated RNAs from the free cytosolic compartment and analyzed the local mtdsRNA expression via qRT-PCR. We found that poly I:C stimulated cells showed increased cytosolic mt-dsRNAs (Fig S2G). We also employed formaldehyde cross-linking and immunoprecipitation (fCLIP) to examine if released mt-dsRNAs interact with PKR. We found that PKR-mtRNA interaction was enhanced upon poly I:C stimulation (Fig S2H). Considering that both poly I:C and mt-dsRNAs can activate PKR, our results suggest that increased cellular mtdsRNAs may amplify the effect of dsRNA-mediated antiviral signaling.

Additionally, we transfected poly I:C to primary SGECs as well as human parotid gland cells (HSY) to show that the observed elevation of mt-dsRNAs was not specific to NS-SV-AC. We found that SGECs obtained from SS patients exhibit higher mt-dsRNA expression levels compared to non-SS sicca patients (Fig S3A), although the sample number is too small to perform statistical analysis. Moreover, we analyzed mt-dsRNA expression upon transfection of dsRNAs and found that primary SGECs from both SS and non-SS sicca subjects (Fig S3B and C) and HSY cells (Fig S3D) all showed increased mt-dsRNA levels. Based on these results, we decided to use NS-SV-AC cells for the rest of our study. 


\section{dsRNA stress downregulates AQP5 and TJC proteins in 3D microenvironment}

The aberrant activation of oral epithelial innate immune response is known to alter the expression and distribution of TJC proteins, which subsequently leads to destruction and secretory dysfunction of salivary and lacrimal glands (Groeger \& Meyle, 2019), resulting in decreased quantity and quality of saliva (Ewert et al, 2010; Zhang et al, 2016). Moreover, the abnormal distribution of AQP5 protein is also evident in the salivary glands of SS patients (Lee et al, 2013; Steinfeld et al, 2001). However, there are limitations to studying the protein expression of TJC and AQP5 in 2D culture due to its weakness in replicating 3D microenvironment. Hence, in order to maintain cell-cell and cell-matrix interactions and reproduce physiological relevance in the context of 3D microenvironment, we employed a poly(2,4,6,7-tetravinyl-2,4,6,8-tetramethyl cyclotetrasiloxane) (pV4D4) functional polymer thin film platform to grow the acinar cells as 3D spheroids (Fig 2A). We chose the pV4D4-coated surface-based spheroid culture method because it can closely mimic in vivo microenvironment by inducing cell-extracellular matrix interactions with enhanced homogeneity in size distribution and circularity of NS-SV-AC compared to conventional options such as Ultra-Low attachment plate (ULA) (Fig S4A and B) (Choi et al, 2018; Yu et al, 2020). Indeed, we confirmed increased expression of TJC proteins (ZO-1 and Occludin) and AQP5 when cells were grown in 3D, and their expressions were too low in the conventional 2D culture system for robust analysis (Fig S4C and D).

With the established 3D culture system, we stimulated NS-SV-AC cells with poly I:C. Consistent with our results in 2D culture, ISGs and PKR phosphorylation were increased, and a decrease in cell viability was evident (Fig 2B-D). In addition, using the 3D system, we found that both ZO-1 and Occludin protein expressions were reduced significantly upon poly I:C stimulation 
(Fig 2E). Moreover, the expression of laminin, a type of extracellular matrix (ECM) protein with reported degradation in SS (Schenke-Layland et al, 2008), also decreased upon poly I:C transfection in 3D culture (Fig S4E). Poly I:C stimulation also led to a dramatic decrease in AQP5 expression (Fig 2F and G). Of note, to analyze AQP5 expression, we used NS-SV-AC cells with stable expression of AQP5-GFP under the constitutive promoter. Last but not least, poly I:C transfection also led to the induction of mt-dsRNAs in our 3D culture system (Fig $2 \mathrm{H})$.

\section{mt-dsRNA induction occurs downstream of JAK1 pathway}

Considering the close association between mt-dsRNA induction and autoimmune signatures of SS, such as TJC and AQP5 downregulation, ISGs induction, and PKR phosphorylation, we continued our investigation to identify the potential upstream pathway responsible for mt-dsRNA induction. We began our study by focusing on JAK-STAT pathway because both type I and II IFNs are known to activate JAK1, and a previous study reported that a selective JAK1 inhibitor is able to reduce IFN-inducible BAFF expression in SGECs (Lee et al, 2018; McInnes et al, 2019).

We first pretreated NS-SV-AC spheroids with upadacitinib, a JAK1 inhibitor, before poly I:C transfection. Our result showed that the pretreatment of upadacitinib significantly reduced the degree of ISGs induction (Fig 3A). Interestingly, the decreased ISGs induction was accompanied by reduced induction of mt-dsRNAs (Fig 3B). Therefore, our data suggest that the JAK1-STAT signaling pathway is responsible for the increased mt-dsRNA expression. 


\section{Effects of M3R ligand Ach are mediated via mt-dsRNAs}

With our in-house developed 3D system, we investigated the relation between mtRNAs and the potential treatments for SS. Currently, patients are often prescribed a set of parasympathomimetic drugs that are known to mimic the effects of Ach. Parasympathomimetic drugs include pilocarpine and cevimeline, which bind to cholinergic (muscarinic) receptors and increase the secretion from the exocrine glands (Lopez-Lopez et al, 2014; Lopez-Pintor et al, 2015; Miranda-Rius et al, 2015). In addition, dysregulated salivation in SS is partly caused by the antagonistic immunoglobulins G (IgGs) against cholinergic receptors of exocrine glands, suppressing the glandular secretory function of M3R in SS (Cha et al, 2006; Passafaro et al, 2010).

First, we examined whether Ach could inhibit poly I:C-triggered ISGs and mt-dsRNA induction. We found that, in the presence of Ach, ISGs induction was significantly attenuated, and this phenomenon was accompanied by decreased induction of mt-dsRNAs (Fig 4A and B). To further validate the importance of mt-dsRNAs in mediating the effect of Ach on ISGs, we depleted mt-dsRNAs using 2- C'-methyladenosine (2-CM), an inhibitor of POLRMT. Pretreatment of 2CM led to a dramatic decrease in the expression of all mt-dsRNAs examined (Fig 4C). We found that the effect of Ach was diminished in mt-dsRNA-depleted cells (Fig 4D), thereby suggesting that the suppression of ISGs by Ach was partly mediated by mt-dsRNAs.

We next analyzed whether the autoantibodies of SS patients could counteract the effect of Ach on suppressing ISG induction. We conducted a similar experiment as above, but this time, we also treated cells with either control human IgG or those pooled from four SS patients (denoted as SS-IgG). We found that SS-IgG resulted in increased ISGs induction and mt-dsRNA expression, thereby lessening the effect of Ach (Fig 4E and F). To further validate the adequacy of human IgGs used as the control, we compared the effect of control human IgG with IgG pooled from anti- 
M3R negative rheumatoid arthritis (RA) patients (denoted as RA-IgG). The data showed indistinguishable differences in ISGs and mt-dsRNAs induction between control IgG and RA-IgG, thereby justifying the use of control human IgG as a negative control for SS-IgG (Fig 4G and H). Overall, our data indicate that SS-IgG may enhance the antiviral response via blockage of M3R interaction with Ach (Fig 4I). Moreover, this provides supporting evidence that the conventional parasympathomimetic drugs for SS may function by modulating cellular mt-dsRNA levels and localization.

\section{Antioxidant RES improves SS-like features through mt-dsRNAs}

In addition to M3R agonists, a previous study reported that administration of RES, a natural polyphenol with antioxidant and anti-inflammatory properties, resulted in increased saliva secretion in NOD mice (Inoue et al, 2016). We asked whether RES may also take part in alleviating dsRNA stress via modulating the levels of mt-dsRNAs. We pretreated NS-SV-AC spheroids with RES $24 \mathrm{~h}$ prior to poly I:C stimulation and examined the effect on downstream response. Through mRNA-seq, we confirmed that RES pretreatment significantly decreased the expression of genes related to the type I IFN signaling pathway (Fig 5A). Twenty-three out of 36 genes relevant to type I IFN showed decreased expression upon RES pretreatment, and we further validated sequencing results for a selected ISGs using qRT-PCR (Fig S5A). Gene ontology (GO) analysis clearly showed that genes related to antiviral immune response (both type I IFN and type II immune responses) were restored upon RES pretreatment (Fig S5B).

Upon RES pretreatment, cell viability was increased by $14.5 \%$ (Fig 5B), and such protective effect may have been translated from reduced ISGs induction and reduced PKR activation (Fig 5C). We then examined the expression of ZO-1, Occludin, and AQP5 and found 
that the expression levels of the aforementioned proteins were all restored to nearly normal (Fig 5D-F). Most interestingly, when we examined the expression of mt-dsRNAs, we found that RES pretreatment decreased the mt-dsRNA levels in poly I:C stimulated cells (Fig 5G). This suggests that the antioxidant RES relieves dsRNA-mediated antiviral signaling partly by protecting mitochondria and preventing the induction and subsequent cytosolic release of mt-dsRNAs.

\section{Targeting mt-dsRNAs ameliorates the autoimmune signatures of SS}

In order to test the accountability of mt-dsRNAs in amplifying antiviral signaling and exacerbating autoimmune characteristics of SS, we analyzed the effect of poly I:C stimulation in mtRNA-depleted cells. Through mRNA-seq, we found that 2-CM pretreatment significantly decreased the expression of genes related to the type I IFN signaling pathway (Fig 6A). Half the number of genes relevant to type I IFN signaling showed reduced levels upon 2-CM pretreatment, and such a result was further confirmed using qRT-PCR (Fig S6A). While poly I:C transfection still induced ISGs, the degree of induction was significantly reduced when mt-dsRNAs were downregulated, suggesting that the increased expression of mt-dsRNAs and their cytosolic export in wild-type cells may aggravate the immune response triggered by poly I:C stimulation. The GO analysis revealed that genes related to oxidative phosphorylation $(4.55 \%)$ and negative regulation of viral genome replication (18.18\%) were partly rescued upon 2-CM pretreatment (Fig S6B). Moreover, similar to RES pretreatment, 2-CM resulted in decreased induction of genes involved in lymphocyte chemotaxis $(3.03 \%)$. Of note, the results of RES pretreatment were strikingly similar to those of 2-CM, further supporting that the effect of RES might be mediated by mtdsRNAs. 
Downregulation of mt-dsRNAs also rescued cell proliferation by approximately $10 \%$ (Fig 6B), and again, this was potentially due to decreased ISGs and reduced phosphorylation of PKR (Fig 6C). We examined the effect of 2-CM pretreatment in the expression of TJCs and AQP5 proteins. 2-CM pretreatment counteracted the effect of poly I:C and almost completely rescued the expression of ZO-1 and Occludin (Fig 6D) as well as AQP5 (Fig 6E and F). Altogether, these results indicate that direct suppression of mt-dsRNAs can rescue autoimmune features of SS.

\section{DISCUSSION}

We here report the important roles of mt-dsRNAs in enhancing autoimmune features of SS. Our data clearly demonstrated that 1) mt-dsRNAs were characteristically elevated in SS patients and that their levels were negatively associated with exocrine dysfunction and sialadenitis; 2) mtdsRNAs were increased in salivary glands of SS mouse model; 3) activation of dsRNA-mediated antiviral signaling led to increased mt-dsRNA expression in SGECs; 4) mt-dsRNA induction was under the control of the JAK1-STAT signaling pathway; 5) the protective effect of M3R ligand Ach was mediated partly by mt-dsRNAs, and SS-IgG negated such protective action of Ach; 6) antioxidant treatment decreased mt-dsRNA expression and autoimmune features of SS; 7) finally, direct downregulation of mt-dsRNAs ameliorated the autoimmune phenotypes of SS (Fig 7).

Our study provides a potential link between mitochondrial damage and the activation of PRRs, which subsequently leads to upregulation of type I IFN. Based on the evidence that mitochondrial dysfunction and accumulation of damaged mitochondria are frequently reported in SS patients (Barrera et al, 2021; Rai et al, 2021), we began our study by stimulating SGECs with poly I:C to yield reactive oxygen species (ROS) stress and disruption of mitochondrial potential (Chen et al, 2016; Harashima et al, 2014). We found that poly I:C stimulation resulted in the 
cytosolic release of mt-dsRNAs, and subsequent PKR phosphorylation and ISG induction. Notably, mt-dsRNA induction and their cytosolic release were essential to augment the antiviral response. Indeed, downregulation of mt-dsRNAs via 2-CM resulted in the attenuation of IFN signature. Considering that mt-dsRNAs can be exported outside the cells and work as extracellular signaling molecules (Kim et al., 2020; Lee et al., 2020b), mt-dsRNAs may play a pathogenic mediator of IFN-derived inflammation in the exocrine glands of SS. Indeed, through various routes, mitochondria are actively involved in the activation of PRRs and innate immune responses, leading to type I IFN and inflammatory responses (Riley \& Tait, 2020).

Our data also indicate that there exists nuclear-mitochondrial communication when cells are under dsRNA stress, leading to the mt-dsRNA accumulation and release. However, genes involved in this process and their molecular mechanism are yet to be determined. Our RNA-seq data provide a potential direction in identifying such factors. Closer examination of DEGs revealed that genes involved in mt-dsRNA stability, such as SLIRP, showed increased expression in poly I:C stimulated cells. Consistent with our hypothesis, SLIRP has been shown to protect the mitochondrial RNAs from degradation (Lagouge et al, 2015), and this may explain how mtdsRNAs were protected from degradation and resulted in rapid accumulation upon poly I:C stimulation. Additionally, the expression of BCL-2 interacting protein 3 (BNIP3), which is responsible for the regulation of mitophagy (Zhang \& Ney, 2009), was increased. Considering that other BCL-2 family proteins - BAX and BAK - are involved in the mitochondrial outer-membrane permeabilization (MOMP) (Pena-Blanco \& Garcia-Saez, 2018), BNIP3 may also play a role in the cytosolic release of mt-dsRNAs through MOMP. Overall, the current study suggests dsRNA stress increases the expression of transcripts that can alter mitochondrial homeostasis and gene induction, 
thereby providing valuable evidence to further investigate the therapeutic potential of targeting mt-dsRNA transcription or cytosolic transport in SS.

Although there is no cure for SS, known medications that are prescribed to alleviate the pains of SS patients are partly dependent on mt-dsRNA modulation. Firstly, we showed that there are clear links between the action mechanism of SS-IgG and mt-dsRNAs. Autoantibodies from SS patients, including anti-M3R autoantibodies, reportedly inhibited the secretory function of exocrine tissues in NOD mice (Robinson et al, 1998) and suppressed cholinergic-induced $\mathrm{Ca}^{2+}$ mobilization and AQP5 trafficking in SGECs (Li et al, 2004). Hence, the long-term administration of approved muscarinic agonist drugs such as pilocarpine was reported to increase M3R expression in the salivary gland (Minagi et al, 2018). Yet, the potential target molecule to diminish such an action mechanism has not been identified. Here, we fill this gap by showing that Ach reduces ISG levels in SGECs by preventing mt-dsRNA induction. The interrelationship of mt-dsRNAs with M3R and SS-IgG can be further investigated to elucidate how the aberrant autoimmune response leads to sicca symptoms. In addition, we also investigated the effect of antioxidant RES, which is known to improve salivary flow in NOD mice. Our data successfully demonstrated that RES ameliorates the degree of mt-dsRNAs induction, thereby yielding protective effects against SSlike autoimmune phenotypes in SGECs. Lastly, we tested the effect of upadacitinib, a JAK1 inhibitor approved for RA treatment, to suggest a potential upstream signaling pathway responsible for mt-dsRNA induction. Our results provide another scientific evidence to support a clinical trial of JAK1 inhibitor in SS. Indeed, due to the ability of JAK inhibitors to suppress ROS- or IFNinduced activation of SGECs, a phase 2 clinical trial is ongoing to assess the safety and efficacy of fligotinib, another JAK1 inhibitor, in SS patients (You et al, 2020). 
There still lies limitations in our study in that we were unable to identify the cellular origin of salivary and tear dsRNAs. As mt-dsRNAs are significantly elevated in the saliva and tear samples of SS patients compared to healthy or non-SS sicca subjects, mt-dsRNAs can be used as a pathophysiological biomarker for earlier diagnosis of SS. Yet, the cellular origin and the mechanism behind how these dsRNAs arrive in saliva or tear still remain unanswered. The majority of mt-dsRNAs may be released from stressed epithelial cells in the oral mucosa or keratoconjunctiva. Still, it will be helpful to find the sources of salivary and tear mt-dsRNAs, since it will provide further hints in developing targeted treatment options for SS.

In sum, our study highlights the importance of mt-dsRNA induction and their cytosolic release in enhancing dsRNA-mediated immune responses and potentiating autoimmune phenotypes in SGECs. This knowledge will have potential implications for the design of targeted therapies to remove accumulated mt-dsRNAs or encourage mt-dsRNA decay to ultimately ameliorate autoimmune characteristics of SS and successfully combat the disease. 


\section{MATERIALS AND METHODS}

\section{Patient saliva and tear samples}

From 42 patients with SS, 31 patients with non-SS sicca, and 16 non-sicca voluntary donors, saliva (34 SS, 23 non-SS sicca, and 16 non-sicca controls) or tears (8 SS and 8 non-SS sicca samples) were collected. SS was classified according to the 2016 American College of Rheumatology/European League Against Rheumatism (ACR/EULAR) classification criteria for primary SS (Shiboski et al, 2017) and non-SS sicca subjects did not fulfill the ACR/EULAR criteria and the 2002 revised American-European Consensus Group criteria (Vitali et al, 2002). Their clinical characteristics are summarized in Table S1.

After application with $50 \mu \mathrm{l}$ of normal saline in the inferior fornix and blinking for $5 \mathrm{sec}$, tear samples were collected from the inferior tear meniscus using glass capillary tubes (Corning). Schirmer test and ocular surface staining scoring were performed to assess tear production and the severity of keratoconjunctivitis sicca, respectively (Whitcher et al, 2010). Saliva samples were collected as described previously (Lee et al, 2010). Unstimulated and stimulated whole salivary flow rates were measured at the time of saliva sample collection. Tear and saliva samples were stored at $-70^{\circ} \mathrm{C}$ until further analysis. All participants provided written informed consent and sample collection was approved by the Institutional Review Board (B-0506/021-004 and B-1806472-301) of Seoul National University Bundang Hospital.

\section{NOD mice SG tissue histology}

All procedures of animal research were provided in accordance with the Laboratory Animals Welfare Act, the Guide for the Care and Use of Laboratory Animals and the Guidelines and Policies for Rodent experiment provided by the IACUC (Institutional Animal Care and Use Committee) in school of medicine, The Catholic University of Korea (Approval numbers: CUMS2019-0255-01). Female NOD/ShiLtJ mice were purchased from The Jackson Laboratory. The animals were maintained under specific pathogen-free conditions from age 8 weeks to age 28 weeks. Mouse submandibular SG tissues were fixed in formalin and embedded in paraffin. 


\section{Cell culture and chemical treatment}

SV40-immortalized NS-SV-AC cell was provided by Professor Masayuki Azuma (Department of Oral Medicine, University of Tokushima Graduate Faculty of Dentistry, Japan) (Azuma et al, 1993). NS-SV-AC human salivary gland acinar cells were grown in Keratinocyte-SFM (Serum free media; Gibco) supplemented with $10 \%(\mathrm{v} / \mathrm{v})$ heat-inactivated fetal bovine serum (FBS; Welgene) and 1\% (v/v) 100x penicillin-streptomycin (Gibco). pGFP-hAQP5-C1 plasmid from Professor Kyung Pyo Park (Seoul National University, South Korea), was used to develop hAQP5expressing NS-SV-AC cell line under the constitutive promoter (100 $\mu \mathrm{g} / \mathrm{ml}$ of G418 selection). All cells were maintained at $37^{\circ} \mathrm{C}$ in a humidified $5 \% \mathrm{CO}_{2}$ incubator. HSY cells were grown in Dulbecco's modified eagle medium (DMEM; Welgene) supplemented with $10 \%(\mathrm{v} / \mathrm{v})$ fetal bovine serum (FBS; Gibco).

To induce dsRNA stress, $20 \mu \mathrm{g} / \mathrm{ml}$ of poly I:C (Sigma Aldrich) was transfected using Lipofectamine 3000 (Thermo Fisher Scientific) for $14 \mathrm{~h}$ following the manufacturer's guide. As for the controlled mock transfection, RNase-free water (Diethyl pyrocarbonate (DEPC)-treated water) was used.

To test the effect of Ach or SS-IgG on mt-dsRNAs induction, $100 \mu \mathrm{M}$ of Ach (Sigma Aldrich) was co-treated with poly I:C transfection. Human IgG was prepared from 4 SS patients with antiM3 muscarinic acetylcholine receptor antibodies (Mona et al, 2020), using the NAb ${ }^{\mathrm{TM}}$ Spin Kits for antibody purification (Thermo Fisher Scientific). IgG was pooled from four individual SS patients $(125 \mu \mathrm{g} /$ patient $)$ and then mixed to the total mass of $500 \mu \mathrm{g}$, then lyophilized. The same method was used to pool IgG from four individual RA patients (125 $\mu \mathrm{g} /$ patient). IgG from human serum (Sigma Aldrich) was used as a control. All IgGs were dissolved in $150 \mathrm{mM} \mathrm{NaCl}$ before treatment. To test the effect of antioxidant on mt-dsRNA, $20 \mu \mathrm{M}$ of RES (Sigma Aldrich) was pretreated for $24 \mathrm{~h}$ prior to poly I:C transfection. To examine the therapeutic effect of JAK1 inhibitor, $1 \mu \mathrm{g} / \mathrm{mL}$ of upadacitinib (MedKoo Biosciences) was treated $1 \mathrm{~h}$ prior to poly I:C transfection. To downregulate mt-dsRNAs expression, $20 \mu \mathrm{M}$ of 2-CM (Santa Cruz Biotechnology) was pretreated $24 \mathrm{~h}$ prior to poly I:C transfection. 


\section{Primary SGEC culture of SS patients}

Patients were diagnosed as having primary SS according to the American-European Consensus Group criteria (Vitali et al., 2002). Informed consent was obtained from all patients according to the principles of the Declaration of Helsinki. Labial salivary gland biopsy tissues were obtained from control patients with sicca symptoms as well as from patients with primary SS. This study was approved by the Institutional Review Board of Seoul St. Mary's Hospital (approval no. KC13ONMI0646). Lip biopsy samples were minced in $1 \mathrm{unit} / \mathrm{ml}$ of Dispase solution (StemCell Technologies) containing $2 \mathrm{mg} / \mathrm{ml}$ of collagenase IV (Gibco) and were then digested with gentle resuspension. Primary salivary gland epithelial cells (SGECs) were cultured in Dulbecco's modified Eagle's Medium-Ham's F-12 (1:3) (Gibco) containing 2.5\% fetal bovine serum (Gibco), $1 \%$ penicillin-streptomycin (Gibco), $0.4 \mu \mathrm{g} / \mathrm{ml}$ hydrocortisone (Sigma), $10 \mathrm{ng} / \mathrm{ml}$ epidermal growth factor (EGF; BioLegend), and $0.5 \mu \mathrm{g} / \mathrm{ml}$ insulin (Gibco). Single-cell suspensions were transferred to bovine type I collagen (PureCol; Advanced BioMatrix)-coated culture dishes.

\section{RNA extraction and quantitative real-time PCR}

To extract total RNAs from cultured cell, TRIzol (Ambion) was added directly to the cell pellet obtained after centrifugation at 10,000 $\times \mathrm{g}$ for $30 \mathrm{sec}$. After precipitation of the extracted nucleic acids, DNase I (TaKaRa) was treated to remove DNA, and purified RNA was reverse transcribed using RevertAid reverse transcriptase (Thermo Fisher Scientific). For RNA extracted from patient samples and 3D spheroids, SuperScript IV Reverse Transcriptase (Invitrogen) was used to synthesize cDNA. For strand-specific qRT-PCR, reverse transcription primers containing CMV promoter sequences were designed to target the specific genes. CDNA was amplified by SYBR Green PCR master mix (Bioline) and qRT-PCR was performed using AriaMx Real-time PCR system (Agilent) and QuantStudio 1 Real-time PCR system (Thermo Fisher Scientific). Primers used in the study are provided in Table S2-S4. To analyze the localization of mtRNAs, cytosolic RNAs were isolated from cell lysates using the subcellular protein fractionation kit (Thermo Fisher Scientific) following the manufacturer's instruction. TRIzol LS (Ambion) was added individually at a 3:1 ratio to the cytosolic fraction to extract the RNAs. The rest of the steps were the same as above. 
To extract RNA from NOD mice SG tissue, $20 \mu \mathrm{m}$-sections were dewaxed using xylene, then dehydrated in ethanol according to a previously published protocol (Ma, 2012). Same reagents were used to synthesize cDNA and mice-specific primers were used for qRT-PCR analysis.

\section{Western Blotting}

Cell lysates were prepared by incubating cells in the lysis buffer $(50 \mathrm{mM}$ Tris- $\mathrm{HCl} \mathrm{pH} 8.0,100$ $\mathrm{mM} \mathrm{KCl}, 0.5 \%$ NP-40, $10 \%$ Glycerol, and 1 mM DTT) supplemented with a protease inhibitor cocktail (Merck) followed by sonication. 30 - $40 \mu \mathrm{g}$ of protein samples were loaded and separated on a 10\% SDS-PAGE gel, and transferred to a PVDF membrane (Merck) using an Amersham semi-dry transfer system. The primary antibodies used in this study were as follows: GFP (Sigma Aldrich; G1544), GAPDH (Santa Cruz Biotechnology; 6C5 sc-32233), pPKR (Abcam, Ab81303), PKR (Cell Signaling Technology, D7F7), and $\beta$-tubulin (Cell Signaling Technology).

\section{Acid phosphatase assay (APH)}

Cells (or spheroids) were centrifuged for $10 \mathrm{~min}$ at $400 \times \mathrm{g}$ for spin down. After washing the pellets twice with DPBS (Welgene, LB001-02), the supernatant was discarded to obtain the final volume of $100 \mu \mathrm{l} .100 \mu \mathrm{l}$ of APH assay buffer (0.1 M sodium acetate, 0.1\% (v/v) Triton X-100 (Promega), $2 \mathrm{mg} / \mathrm{ml}$ Immunopure PNPP (Sigma Aldrich) in deionized/distilled water) was added to each well and incubated for $90 \mathrm{~min}$ at $37^{\circ} \mathrm{C}$ with $5 \% \mathrm{CO}_{2}$. After incubation, $10 \mu \mathrm{l}$ of $1 \mathrm{~N} \mathrm{NaOH}$ was added, and absorption at $405 \mathrm{~nm}$ was measured on a microplate reader (BioTek). For each experiment, cell viability was normalized to the data from the control group without poly I:C transfection.

\section{Immunocytochemistry and RNA single-molecule fluorescent in situ hybridization}

Spheroids from pV4D4-coated plates were transferred to a $1.5 \mathrm{ml}$ tube, and analysis for 2D culture was performed on a confocal dish (SPL). Cells were fixed in 4\% (w/v) paraformaldehyde for 15 min at room temperature. Fixed cells were then permeabilized with $0.3 \%$ (w/v) Triton X-100 (Sigma Aldrich) in Dulbecco's phosphate-buffered saline (DPBS) for $10 \mathrm{~min}$ at room temperature and blocked in 3\% bovine serum albumin (BSA100; Bovogen) for $1 \mathrm{~h}$. Cells were incubated with primary antibodies diluted in $1 \% \mathrm{BSA}$ at a $1: 100$ ratio overnight at $4^{\circ} \mathrm{C}$. The primary antibodies used in this study include: Occludin (Invitrogen; OC-3F10) and ZO-1 (Cell Signaling Technology; 8193S). Cells were then washed with DPBS and incubated with Alexa fluor 488-conjugated anti- 
mouse secondary antibody (Thermo Fisher Scientific; A31570) and Alexa fluor 555 conjugated anti-mouse secondary antibody (Thermo Fisher Scientific; A-21202) diluted at a 1:1000 ratio for $45 \mathrm{~min}$ at room temperature. DAPI was incubated for $5 \mathrm{~min}$. All washing steps for spheroids include centrifugation (5 min, $200 \mathrm{~g}$ ) and supernatant discarding. Fluorescent images were obtained using a confocal laser-scanning microscope (LSM 880, Carl Zeiss). The fluorescence intensity per area was quantified as integrated density divided by the area of each spheroid and then normalized to the control without poly I:C transfection.

To visualize heavy and light strands of mt-Nd5 mtRNA from NOD mice SG tissue, fivemicrometer sections were dewaxed using xylene, then dehydrated in ethanol for staining with RNAscope RNA fluorescent in situ hybridization (ACD) probes. The pretreatment, hybridization, and signal amplification were done following the manufacturer's instructions. Zeiss LSM 780 confocal microscope using 63x $(\mathrm{NA}=1.40)$ objectives was used for visualization.

\section{PKR fCLIP}

To prepare PKR antibody-conjugated beads, protein A beads (Thermo Fisher Scientific) were incubated with PKR antibody (Cell Signaling Technology) in the fCLIP lysis buffer (20 mM Tris$\mathrm{HCl}, \mathrm{pH} 7.5,15 \mathrm{mM} \mathrm{NaCl}, 10 \mathrm{mM}$ EDTA, $0.5 \%$ NP-40, 0.1\% Triton X-100, 0.1\% SDS, and 0.1\% sodium deoxycholate) for $3 \mathrm{~h}$ at $4^{\circ} \mathrm{C}$ after adjusting $\mathrm{NaCl}$ concentration to $150 \mathrm{mM}$. Harvested cells were fixed with $0.1 \%(\mathrm{w} / \mathrm{v})$ paraformaldehyde (Sigma Aldrich) for $10 \mathrm{~min}$ and immediately quenched by adjusting glycine (Bio-basic) concentration to $250 \mathrm{mM}$ for an additional $10 \mathrm{~min}$ at room temperature. The cross-linked cells were lysed using the fCLIP lysis buffer for 10 min on ice and then sonicated. The $\mathrm{NaCl}$ concentration of the lysate was adjusted to $150 \mathrm{mM}$, and cell debris was separated by centrifugation. The lysate was added to the PKR antibody-conjugated beads and incubated for $3 \mathrm{~h}$ at $4^{\circ} \mathrm{C}$. The beads were washed 4 times with the fCLIP wash buffer (20 mM Tris-HCl, pH 7.5, 150 mM NaCl, 10 mM EDTA, 0.1\% NP-40, 0.1\% SDS, 0.1\% Triton $\mathrm{X}-100$, and $0.1 \%$ sodium deoxycholate) and PKR-dsRNA complex was eluted from the beads by incubating in the elution buffer $(200 \mathrm{mM}$ Tris-HCl, pH 7.4, $100 \mathrm{mM} \mathrm{NaCl}, 20 \mathrm{mM}$ EDTA, 2\% $\mathrm{SDS}$, and $7 \mathrm{M} \mathrm{Urea)} \mathrm{for} 3 \mathrm{~h}$ at $25^{\circ} \mathrm{C}$. The eluate was treated with $2 \mathrm{mg} / \mathrm{ml}$ proteinase $\mathrm{K}$ (Sigma Aldrich) for overnight at $65^{\circ} \mathrm{C}$. RNA was purified using acid-phenol:Chloroform $\mathrm{pH} 4.5$ (Thermo Fisher Scientific). 


\section{Polymer thin films via the initiated chemical vapor deposition process}

A 300 nm-thick pV4D4 film was deposited directly onto tissue culture polystyrene (TCPS) via the initiated chemical vapor deposition (iCVD) process. TCPS was first placed on the stage of a custom-built iCVD chamber. V4D4 (97\%; Jihyunchem Co.) was heated to $70^{\circ} \mathrm{C}$ for monomer vaporization. The vaporized monomer V4D4, and the initiator, tert-butyl peroxide (TBPO; 95\%; Sigma Aldrich) were introduced into the iCVD chamber at flow rates of 1.94 and $0.744 \mathrm{sccm}$, respectively. The pressure of the chamber was set to 260 mTorr. To decompose TBPO to generate radicals, the filament temperature was heated to $200^{\circ} \mathrm{C}$. During the deposition process, the stage was maintained to $38.5^{\circ} \mathrm{C}$ for the adsorption of monomers.

\section{D spheroid culture on a pV4D4-coated plate}

Cells were seeded at the density of $1 \times 10^{5}$ cells $/ \mathrm{ml}$ on a pV4D4-coated plate and cultured in the same media described above and maintained at $37^{\circ} \mathrm{C}$ in a humidified $5 \% \mathrm{CO}_{2}$ incubator. The spheroids formed on the pV4D4-coated plate were imaged at 24, 48, and $72 \mathrm{~h}$ after seeding by phase-contrast microscopy (Eclipse Ti-U; Nikon). For the live-imaging of spheroid formation, cells were monitored using an optical microscope (LumaScope 620 System; Etaluma). Images were acquired every 2 min for $24 \mathrm{~h}$ with a $10 \mathrm{X}$ magnification.

\section{Data analysis of mRNA-seq data}

Read files were quality checked with FastQC. Reads were aligned to the human genome (hg38) using Hisat2 (ver 2.1.0) (Kim et al, 2019). The aligned reads were quantified with StringTie (Pertea et al, 2015) using human gene annotation file version 32 from Gencode as the index. Differentially expressed genes were searched with DESeq2 (Love et al, 2014).

\section{Differentially expressed gene (DEG) analysis}

For DEG analysis, DESeq2 analysis (Love et al., 2014) was performed three times separately in each of DMSO, 2-CM, and RES-pretreated groups. Within each group, raw counts of two biological replicates with poly I:C transfection were compared against the raw counts of two replicates with the control group (i.e. DEPC transfection). The counts were normalized by DESeq2 
during analysis. The analysis yielded three separate lists of differentially expressed genes, and the genes were ordered by the lowest adjusted p-values for further analysis.

\section{Gene ontology (GO) analysis of differentially expressed RNAs}

Among genes that show $\log 2$ fold change with p-values less than 0.05, the values of DMSO (i.e. control) were subtracted from those of RES or 2-CM-pretreated RNAs to evaluate the degree of rescue in the induction of gene expression. Top 200 of the genes that show rescue effects were assembled into a gene list for GO analysis. GO analysis was done using the ClueGo software (Cytoscape, v. 3.7.1) (Bindea et al, 2009).

\section{Statistical Analysis}

Quantitative RT-PCR data and APH assay results were analyzed using the one-tailed Student's ttest. All data were biologically replicated at least three times. The error bars indicate the standard error of the mean. p-values $\leq 0.05$ were regarded as statistically significant. * denotes p-values $\leq$ $0.05, * *$ is $\mathrm{p}$-values $\leq 0.01$, and $* * *$ is $\mathrm{p}$-values $\leq 0.001$.

Online supplemental material: Online supplemental material is available.

Data and materials availability: All data needed to evaluate the conclusions in the paper are present in the paper and/or the Supplementary Materials. Additional data related to this paper may be requested from the first and/or corresponding author/s. Sequencing data that support the findings of this study are available in the GEO database under the accession number GSE168148. All other relevant datasets used and/or analyzed during the current study are available within the article and its Supplementary Materials files or from the corresponding authors on reasonable request. The computational pipeline used in this study is open-sourced and available at: https://daehwankimlab.github.io/hisat2/ , https://ccb.jhu.edu/software/stringtie/ , and http://www.bioconductor.org/packages/release/bioc/html/DESeq2.html.

Acknowledgments: We thank all members of the Lee, Im, and Kim laboratory for helpful discussion and comments on the paper. 
Funding: This work was supported by the Ministry of Health \& Welfare (HI21C1501, to Y.K.), KAIST-SNUBH End-Run Project (N11180151, to Y.K. and No. 16-2018-003 to Y.J.L.), Technology Innovation Program (No. 20008777, to S. I.) by the Ministry of Trade, Industry and Energy (MOTIE, Korea), and the Sjögren's Foundation and NIH/NIAIMS (AR079693, to S.C.).

Author contributions: J. Yoon, M. Lee, YJ. Lee, SG. Lim, and Y. Kim designed the study and analysis. Experiments were performed by J. Yoon, and M. Lee performed imaging and cell viability analysis of 3D spheroids. YJ. Lee provided the saliva samples. A.A. Ali analyzed the saliva samples and performed the initial characterization of 3D spheroids. YR. Oh and YS. Choi collected and prepared patient samples and developed an hAQP5-expressing NS-SV-AC cell line. N. Lee processed the mRNA-seq raw data file for DEG analysis. SG. Jang and S. Kwok provided the NOD mouse tissue samples and primary cells of SS patients. J. Yoon and S. Kim analyzed the salivary gland tissues of NOD mice. JY. Hyon and S. Cha designed the project with YJ. Lee. The study was supervised by Y. Kim, YJ. Lee, and SG. Im. J. Yoon and Y. Kim wrote the manuscript with contributions from N. Ahamad, S. Cha, and S. Kim. All authors subsequently reviewed and edited the manuscript.

Competing interests: S.G.I. is a co-inventor of the polymeric thin film-based 3D culture used in this study and is currently filing a patent for it. The authors declare no competing interests. 


\section{References}

Ahmad S, Mu X, Yang F, Greenwald E, Park JW, Jacob E, Zhang CZ, Hur S (2018) Breaching Self-Tolerance to Alu Duplex RNA Underlies MDA5-Mediated Inflammation. Cell 172: 797810 e 713

Azuma M, Tamatani T, Kasai Y, Sato M (1993) Immortalization of normal human salivary gland cells with duct-, myoepithelial-, acinar-, or squamous phenotype by transfection with SV40 orimutant deoxyribonucleic acid. Lab Invest 69: 24-42

Barrera MJ, Aguilera S, Castro I, Carvajal P, Jara D, Molina C, Gonzalez S, Gonzalez MJ (2021) Dysfunctional mitochondria as critical players in the inflammation of autoimmune diseases: Potential role in Sjo \& uml;gren's syndrome. Autoimmunity Reviews 20 Bikker A, van Woerkom JM, Kruize AA, Wenting-van Wijk M, de Jager W, Bijlsma JW, Lafeber FP, van Roon JA (2010) Increased expression of interleukin-7 in labial salivary glands of patients with primary Sjogren's syndrome correlates with increased inflammation. Arthritis Rheum 62: 969-977

Bindea G, Mlecnik B, Hackl H, Charoentong P, Tosolini M, Kirilovsky A, Fridman WH, Pages F, Trajanoski Z, Galon J (2009) ClueGO: a Cytoscape plug-in to decipher functionally grouped gene ontology and pathway annotation networks. Bioinformatics 25: 1091-1093

Cha S, Singson E, Cornelius J, Yagna JP, Knot HJ, Peck AB (2006) Muscarinic acetylcholine type-3 receptor desensitization due to chronic exposure to Sjogren's syndrome-associated autoantibodies. J Rheumatol 33: 296-306

Chen H, Wang DL, Liu YL (2016) Poly (I:C) transfection induces mitochondrial-mediated apoptosis in cervical cancer. Mol Med Rep 13: 2689-2695

Choi M, Yu SJ, Choi Y, Lee HR, Lee E, Lee E, Lee Y, Song J, Son JG, Lee TG et al (2018) Polymer Thin Film-Induced Tumor Spheroids Acquire Cancer Stem Cell-like Properties. Cancer Res 78: 6890-6902

Cole MB, Quach H, Quach D, Baker A, Taylor KE, Barcellos LF, Criswell LA (2016) Epigenetic Signatures of Salivary Gland Inflammation in Sjogren's Syndrome. Arthritis Rheumatol 68: 2936-2944

Crow MK, Olferiev M, Kirou KA (2019) Type I Interferons in Autoimmune Disease. Annu Rev Pathol 14: 369-393

Deshmukh US, Nandula SR, Thimmalapura PR, Scindia YM, Bagavant H (2009) Activation of innate immune responses through Toll-like receptor 3 causes a rapid loss of salivary gland function. J Oral Pathol Med 38: 42-47

Dhir A, Dhir S, Borowski LS, Jimenez L, Teitell M, Rotig A, Crow YJ, Rice GI, Duffy D, Tamby C et al (2018) Mitochondrial double-stranded RNA triggers antiviral signalling in humans. Nature 560: 238-242

Ewert P, Aguilera S, Alliende C, Kwon YJ, Albornoz A, Molina C, Urzua U, Quest AF, Olea N, Perez $\mathrm{P}$ et al (2010) Disruption of tight junction structure in salivary glands from Sjogren's syndrome patients is linked to proinflammatory cytokine exposure. Arthritis Rheum 62: 12801289

Greenwell-Wild T, Moutsopoulos NM, Gliozzi M, Kapsogeorgou E, Rangel Z, Munson PJ, Moutsopoulos HM, Wahl SM (2011) Chitinases in the salivary glands and circulation of patients with Sjogren's syndrome: macrophage harbingers of disease severity. Arthritis Rheum 63: 31033115

Groeger S, Meyle J (2019) Oral Mucosal Epithelial Cells. Front Immunol 10: 208 
Guo Z, Li Y, Ding SW (2019) Small RNA-based antimicrobial immunity. Nat Rev Immunol 19: 31-44

Harashima N, Minami T, Uemura H, Harada M (2014) Transfection of poly(I:C) can induce reactive oxygen species-triggered apoptosis and interferon- $\beta$-mediated growth arrest in human renal cell carcinoma cells via innate adjuvant receptors and the 2-5A system. Molecular cancer 13: $217-217$

Hu S, Zhou M, Jiang J, Wang J, Elashoff D, Gorr S, Michie SA, Spijkervet FK, Bootsma H, Kallenberg CG et al (2009) Systems biology analysis of Sjogren's syndrome and mucosaassociated lymphoid tissue lymphoma in parotid glands. Arthritis Rheum 60: 81-92 Huijser E, Versnel MA (2021) Making Sense of Intracellular Nucleic Acid Sensing in Type I Interferon Activation in Sjogren's Syndrome. J Clin Med 10

Inoue H, Kishimoto A, Ushikoshi-Nakayama R, Hasaka A, Takahashi A, Ryo K, Muramatsu T, Ide F, Mishima K, Saito I (2016) Resveratrol improves salivary dysfunction in a non-obese diabetic (NOD) mouse model of Sjogren's syndrome. J Clin Biochem Nutr 59: 107-112 Jin JO, Shinohara Y, Yu Q (2013) Innate immune signaling induces interleukin-7 production from salivary gland cells and accelerates the development of primary Sjogren's syndrome in a mouse model. PLoS One 8: e77605

Jonsson MV, Delaleu N, Brokstad KA, Berggreen E, Skarstein K (2006) Impaired salivary gland function in nod mice - Association with changes in cytokine profile but not with salivary gland histopathology. Arthritis Rheum-Us 54: S707-S707

Kim D, Paggi JM, Park C, Bennett C, Salzberg SL (2019) Graph-based genome alignment and genotyping with HISAT2 and HISAT-genotype. Nat Biotechnol 37: 907-915

Kim S, Lee K, Choi YS, Ku J, Lee YJ, Kim Y (2020) Mitochondrial dsRNAs activate PKR and TLR3 to promote chondrocyte degeneration in osteoarthritis. bioRxiv: 2020.2006.2017.156323

Kim Y, Park J, Kim S, Kim M, Kang MG, Kwak C, Kang M, Kim B, Rhee HW, Kim VN (2018) PKR Senses Nuclear and Mitochondrial Signals by Interacting with Endogenous DoubleStranded RNAs. Mol Cell 71: 1051-1063 e1056

Kiripolsky J, Kramer JM (2018) Current and Emerging Evidence for Toll-Like Receptor Activation in Sjogren's Syndrome. J Immunol Res 2018: 1246818

Kroese FG, Abdulahad WH, Haacke E, Bos NA, Vissink A, Bootsma H (2014) B-cell

hyperactivity in primary Sjogren's syndrome. Expert Rev Clin Immunol 10: 483-499

Lagouge M, Mourier A, Lee HJ, Spahr H, Wai T, Kukat C, Silva Ramos E, Motori E, Busch JD, Siira S et al (2015) SLIRP Regulates the Rate of Mitochondrial Protein Synthesis and Protects LRPPRC from Degradation. PLoS Genet 11: e1005423

Lee BH, Gauna AE, Perez G, Park YJ, Pauley KM, Kawai T, Cha S (2013) Autoantibodies against muscarinic type 3 receptor in Sjogren's syndrome inhibit aquaporin 5 trafficking. PLoS

One 8: e53113

Lee H, Fenster RJ, Pineda SS, Gibbs WS, Mohammadi S, Davila-Velderrain J, Garcia FJ, Therrien M, Novis HS, Gao F et al (2020a) Cell Type-Specific Transcriptomics Reveals that Mutant Huntingtin Leads to Mitochondrial RNA Release and Neuronal Innate Immune Activation. Neuron 107: 891-908 e898

Lee J, Lee J, Kwok SK, Baek S, Jang SG, Hong SM, Min JW, Choi SS, Lee J, Cho ML et al (2018) JAK-1 Inhibition Suppresses Interferon-Induced BAFF Production in Human Salivary Gland: Potential Therapeutic Strategy for Primary Sjogren's Syndrome. Arthritis Rheumatol 70: 2057-2066 
Lee JH, Shim YR, Seo W, Kim MH, Choi WM, Kim HH, Kim YE, Yang K, Ryu T, Jeong JM et al (2020b) Mitochondrial Double-Stranded RNA in Exosome Promotes Interleukin-17 Production Through Toll-Like Receptor 3 in Alcohol-associated Liver Injury. Hepatology 72: 609-625

Lee YJ, Scofield RH, Hyon JY, Yun PY, Lee HJ, Lee EY, Lee EB, Song YW (2010) Salivary chemokine levels in patients with primary Sjogren's syndrome. Rheumatology (Oxford) 49: 1747-1752

Li JC, Ha YM, Ku NY, Choi SY, Lee SJ, Oh SB, Kim JS, Lee JH, Lee EB, Song YW et al (2004) Inhibitory effects of autoantibodies on the muscarinic receptors in Sjogren's syndrome. Laboratory Investigation 84: 1430-1438

Lopez-Lopez J, Jane Salas E, Chimenos Kustner E (2014) [Prognosis and treatment of dry mouth. Systematic review]. Med Clin (Barc) 142: 119-124

Lopez-Pintor RM, Fernandez Castro M, Hernandez G (2015) Oral involvement in patients with primary Sjogren's syndrome. Multidisciplinary care by dentists and rheumatologists. Reumatol Clin 11: 387-394

Love MI, Huber W, Anders S (2014) Moderated estimation of fold change and dispersion for RNA-seq data with DESeq2. Genome Biol 15: 550

Ma Z (2012) Total RNA Extraction from Formalin-Fixed, Paraffin-Embedded (FFPE) Blocks. Bio-protocol 2: e161

Maria NI, Steenwijk EC, AS IJ, van Helden-Meeuwsen CG, Vogelsang P, Beumer W, Brkic Z, van Daele PL, van Hagen PM, van der Spek PJ et al (2017) Contrasting expression pattern of RNA-sensing receptors TLR7, RIG-I and MDA5 in interferon-positive and interferon-negative patients with primary Sjogren's syndrome. Ann Rheum Dis 76: 721-730

McInnes IB, Byers NL, Higgs RE, Lee J, Macias WL, Na S, Ortmann RA, Rocha G, Rooney TP, Wehrman T et al (2019) Comparison of baricitinib, upadacitinib, and tofacitinib mediated regulation of cytokine signaling in human leukocyte subpopulations. Arthritis Res Ther 21: 183 Min HK, Moon SJ, Park KS, Kim KJ (2019) Integrated systems analysis of salivary gland transcriptomics reveals key molecular networks in Sjogren's syndrome. Arthritis Res Ther 21: 294

Minagi HO, Ikai K, Araie T, Sakai M, Sakai T (2018) Benefits of long-term pilocarpine due to increased muscarinic acetylcholine receptor 3 in salivary glands. Biochem Bioph Res Co 503: 1098-1102

Miranda-Rius J, Brunet-Llobet L, Lahor-Soler E, Farre M (2015) Salivary Secretory Disorders, Inducing Drugs, and Clinical Management. Int J Med Sci 12: 811-824

Mona M, Mondello S, Hyon JY, Saleh W, Han K, Lee HJ, Ha YJ, Kang EH, Lee YJ, Cha S (2020) Clinical usefulness of anti-muscarinic type 3 receptor autoantibodies in patients with primary Sjogren's syndrome. Clin Exp Rheumatol

Pagano G, Castello G, Pallardo FV (2013) Sjogren's syndrome-associated oxidative stress and mitochondrial dysfunction: prospects for chemoprevention trials. Free Radic Res 47: 71-73 Park YS, Gauna AE, Cha S (2015) Mouse Models of Primary Sjogren's Syndrome. Curr Pharm Des 21: 2350-2364

Passafaro D, Reina S, Sterin-Borda L, Borda E (2010) Cholinergic autoantibodies from primary Sjogren's syndrome modulate submandibular gland $\mathrm{Na}+\mathrm{K}+-\mathrm{ATPase}$ activity via prostaglandin E2 and cyclic AMP. Eur J Oral Sci 118: 131-138

Pena-Blanco A, Garcia-Saez AJ (2018) Bax, Bak and beyond - mitochondrial performance in apoptosis. FEBS J 285: 416-431 
Pertea M, Pertea GM, Antonescu CM, Chang TC, Mendell JT, Salzberg SL (2015) StringTie enables improved reconstruction of a transcriptome from RNA-seq reads. Nat Biotechnol 33: 290-295

Qin B, Wang J, Yang Z, Yang M, Ma N, Huang F, Zhong R (2015) Epidemiology of primary Sjogren's syndrome: a systematic review and meta-analysis. Ann Rheum Dis 74: 1983-1989

Rai P, Janardhan KS, Meacham J, Madenspacher JH, Lin WC, Karmaus PWF, Martinez J, Li QZ, Yan M, Zeng J et al (2021) IRGM1 links mitochondrial quality control to autoimmunity. Nat Immunol 22: 312-321

Riley JS, Tait SW (2020) Mitochondrial DNA in inflammation and immunity. EMBO Rep 21: e49799

Robinson CP, Brayer J, Yamachika S, Esch TR, Peck AB, Stewart CA, Peen E, Jonsson R, Humphreys-Beher M (1998) Transfer of human serum IgG to nonobese diabetic Ig mu(null) mice reveals a role for autoantibodies in the loss of secretory function of exocrine tissues in Sjogren's syndrome. Arthritis Rheum-Us 41: S354-S354

Ryo K, Yamada H, Nakagawa Y, Tai Y, Obara K, Inoue H, Mishima K, Saito I (2006) Possible involvement of oxidative stress in salivary gland of patients with Sjogren's syndrome.

Pathobiology 73: 252-260

Schenke-Layland K, Xie JS, Angelis E, Starcher B, Wu KJ, Riemann I, MacLellan WR, HammAlvarez SF (2008) Increased degradation of extracellular matrix structures of lacrimal glands implicated in the pathogenesis of Sjogren's syndrome. Matrix Biology 27: 53-66

Shiboski CH, Shiboski SC, Seror R, Criswell LA, Labetoulle M, Lietman TM, Rasmussen A, Scofield H, Vitali C, Bowman SJ et al (2017) 2016 American College of

Rheumatology/European League Against Rheumatism Classification Criteria for Primary

Sjogren's Syndrome: A Consensus and Data-Driven Methodology Involving Three International Patient Cohorts. Arthritis Rheumatol 69: 35-45

Stefanski AL, Tomiak C, Pleyer U, Dietrich T, Burmester GR, Dorner T (2017) The Diagnosis and Treatment of Sjogren's Syndrome. Dtsch Arztebl Int 114: 354-361

Steinfeld S, Cogan E, King LS, Agre P, Kiss R, Delporte C (2001) Abnormal distribution of aquaporin-5 water channel protein in salivary glands from Sjogren's syndrome patients. Lab Invest 81: 143-148

Vitali C, Bombardieri S, Jonsson R, Moutsopoulos HM, Alexander EL, Carsons SE, Daniels TE, Fox PC, Fox RI, Kassan SS et al (2002) Classification criteria for Sjogren's syndrome: a revised version of the European criteria proposed by the American-European Consensus Group. Ann Rheum Dis 61: 554-558

Whitcher JP, Shiboski CH, Shiboski SC, Heidenreich AM, Kitagawa K, Zhang S, Hamann S, Larkin G, McNamara NA, Greenspan JS et al (2010) A simplified quantitative method for assessing keratoconjunctivitis sicca from the Sjogren's Syndrome International Registry. Am J Ophthalmol 149: 405-415

You H, Xu D, Zhao J, Li J, Wang Q, Tian X, Li M, Zeng X (2020) JAK Inhibitors: Prospects in Connective Tissue Diseases. Clin Rev Allergy Immunol 59: 334-351

Yu SJ, Choi G, Cho Y, Lee M, Cho Y, Shin JH, Lee E, Im SG (2020) Three-Dimensional Spheroid Culture on Polymer-Coated Surface Potentiate Stem Cell Functions via Enhanced CellExtracellular Matrix Interactions. Acs Biomater Sci Eng 6: 2240-2250

Zhang J, Ney PA (2009) Role of BNIP3 and NIX in cell death, autophagy, and mitophagy. Cell Death Differ 16: 939-946 
Zhang LW, Cong X, Zhang Y, Wei T, Su YC, Serrao AC, Brito AR, Jr., Yu GY, Hua H, Wu LL (2016) Interleukin-17 Impairs Salivary Tight Junction Integrity in Sjogren's Syndrome. J Dent Res 95: 784-792

Zhou J, Jin JO, Du J, Yu Q (2015) Innate Immune Signaling Induces IL-7 Production, Early Inflammatory Responses, and Sjogren's-Like Dacryoadenitis in C57BL/6 Mice. Invest Ophthalmol Vis Sci 56: 7831-7838 


\section{Figure Legends}

Fig. 1. mt-dsRNAs are elevated in saliva and tears of SS patients as well as salivary gland tissues of aged NOD mice. (A) The experimental scheme. To distinguish between mt-dsRNAs from the heavy and light strands, strand-specific reverse transcription was used to synthesize cDNAs. (B) The relative RNA expression levels of both heavy $(\mathrm{H})$ and light (L) strands in saliva among non-sicca control, non-SS sicca and SS patients. (C) The relative RNA expression levels in tears between non-SS sicca and SS patients. All Cq values are relative to GAPDH mRNA, then normalized to non-sicca and non-SS sicca controls. (D) Expression levels of five SS-associated mt-dsRNAs in the salivary gland of NOD mice. All Cq values are relative to Actb mRNA, then normalized to 8-week old NOD mouse samples. Results are presented as the mean \pm SEM of three independent samples. (E) Representative fluorescence images of NOD mouse salivary gland tissues with mt-Nd5 light $(N d 5 L)$ and heavy strand $(N d 5 H)$ transcripts. $(\mathbf{F})$ The negative control probe yielded no signal. For $(\mathbf{E})$ and $(\mathbf{F})$, the scale bar represents $50 \mu \mathrm{m}$.

Fig. 2. dsRNA stimulation induces autoimmune features of SS and mt-dsRNA expression in SGECs spheroids. (A) A schematic representation of 3D culture by depositing V4D4 monomers via iCVD process. (B) The relative mRNA expression of indicated ISGs in poly I:C transfected NS-SV-AC spheroids. RNA was isolated and the indicated ISGs expressions were quantified by qRT-PCR. Shown are normalized $\log _{2}$ fold enrichment of three independent experiments with error bars denoting SEM. All Cq values are relative to ACTB mRNA, then normalized to Mock transfected control. (C) Western blot analysis of PKR phosphorylation upon poly I:C transfection in 3D system. (D) APH assay showing cell viability upon poly I:C transfection. Shown are four independent experiments with error bars denoting SEM. (E-G) Poly I:C transfection led to 
decreased TJC and AQP5-GFP expressions. (E) Representative fluorescence images of ZO-1 and Occludin upon poly I:C transfection. (F) Representative fluorescence images of AQP5-GFP upon poly I:C transfection. The fluorescence intensities were quantified as the mean of three independent experiments and normalized to the Mock transfection control group. For $(\mathbf{E})$ and $(\mathbf{F})$, the scale bar represents $100 \mu \mathrm{m}$. (G) Western blot analysis of AQP5-GFP expression upon poly I:C transfection. (H) The relative mt-dsRNA expression in poly I:C transfected NS-SV-AC spheroids. RNA was isolated and mt-dsRNA expressions were quantified by qRT-PCR. Shown are normalized $\log _{2}$ fold enrichment of three independent experiments with error bars denoting SEM. All Cq values are relative to $A C T B$ mRNA, then normalized to Mock transfected control. All statistical significances were calculated using one-tailed Student's t-tests, *p $\leq 0.05$, **p $\leq$ 0.01 , and $* * * \mathrm{p} \leq 0.001$

Fig. 3. mt-dsRNA induction occurs downstream of the JAK1 signaling pathway. NS-SV-AC spheroids were pretreated with upadacitinib $1 \mathrm{~h}$ prior to poly I:C stimulation. From the isolated RNAs, the expression levels of eight ISGs (A), and mt-dsRNAs (B) were quantified by qRT-PCR. All Cq values are relative to $A C T B$ mRNA. For upadacitinib-untreated control (CTRL), values of poly I:C-transfected samples were normalized to Mock-transfected samples. The same normalization was carried out for upadacitinib-treated samples. The degree of induction with or without upadacitinib pretreatment was then compared by computing the ratio between CTRL and upadacitinib-treated poly I:C-transfected samples. Shown are three independent experiments with error bars denoting SEM. All statistical significances were calculated using one-tailed Student's ttests, $* \mathrm{p} \leq 0.05, * * \mathrm{p} \leq 0.01$, and $* * * \mathrm{p} \leq 0.001$ 
Fig. 4. M3R ligand Ach prevents mt-dsRNA induction to yield the protective effects against SS-like autoimmune phenotypes. NS-SV-AC spheroids were stimulated with poly I:C in the presence or the absence of $100 \mu \mathrm{M}$ Ach. From the isolated RNAs, the expression levels of eight ISGs (A), and mt-dsRNAs (B) were quantified by qRT-PCR. All Cq values are relative to ACTB mRNA. For the control group, values of poly I:C-transfected samples were normalized to Mocktransfected samples. The same normalization was carried out for samples transfected with poly I:C in the presence of Ach. The degree of induction in the presence or the absence of Ach was then compared by computing the ratio between CTRL and Ach-treated poly I:C-transfected samples. (C) NS-SV-AC spheroids were treated with 2-CM, then the expression levels of mt-dsRNAs were measured by qRT-PCR. Shown are six independent experiments with SEM. (D) The ratio of ISGs induction upon poly I:C transfection between Ach-treated and control samples in 2-CM-pretreated cells. (E-F) NS-SV-AC spheroids were stimulated with poly I:C in the presence of $100 \mu \mathrm{M}$ Ach and $500 \mu \mathrm{g}$ of control IgG or SS-IgG. From the isolated RNAs, the expression levels of eight ISGs (E), and mt-dsRNAs (F) were quantified by qRT-PCR. The degree of induction upon poly I:C transfection in the presence of Ach with or without SS-IgG was compared by computing the ratio between CTRL and SS-IgG-treated poly I:C transfected samples. (G-H) The effect of control human IgG was compared with RA-IgG. The degree of ISGs $(\mathbf{G})$ and mt-dsRNA (H) induction upon poly I:C stimulation in the presence of Ach with either control IgG or RA-IgG was compared by computing the ratio between CTRL and RA-IgG-treated poly I:C transfected samples. (I) A schematic representation of intracellular Ach interacting with M3R to prevent mt-dsRNA release to the cytosol. Unless mentioned otherwise, the shown graph represents three independent experiments with error bars denoting SEM. All statistical significances were calculated using onetailed Student's t-tests, $* \mathrm{p} \leq 0.05, * * \mathrm{p} \leq 0.01$, and $* * * \mathrm{p} \leq 0.001$. 
Fig. 5. Antioxidant improves SS-like features partly by downregulating mt-dsRNA expression. NS-SV-AC spheroids were pretreated with $20 \mu \mathrm{M}$ RES $24 \mathrm{~h}$ prior to poly I:C stimulation. (A) Heatmap of mRNA sequencing results for type I IFN signaling pathway genes. The two columns represent $\log _{2}$ fold changes with or without RES pretreatment. (B) APH assay showing the effect of RES on cell viability. Shown are four independent experiments with error bars denoting SEM. (C) Western blot analysis of PKR phosphorylation upon poly I:C stimulation with or without RES pretreatment. (D) Representative fluorescence images of ZO-1 and Occludin expression. Similarly, AQP5-GFP expression upon poly I:C stimulation with or without RES pretreatment was analyzed by $(\mathbf{E})$ western blot and $(\mathbf{F})$ immunocytochemistry. The fluorescence intensities in (D) and (F) were quantified as the mean of three independent experiments and normalized to the Mock transfection control group. The scale bar represents $100 \mu \mathrm{m}$. (G) Expression levels of mt-dsRNAs in the poly I:C-stimulated NS-SV-AC with or without RES pretreatment. All $\mathrm{Cq}$ values are relative to $A C T B$ mRNA. For the control group, values of poly I:C-stimulated samples were normalized to Mock-transfected samples. The same normalization was carried out for RES-pretreated samples. The degree of induction with or without RES pretreatment was then compared by computing the ratio between CTRL and RES-pretreated poly I:C-stimulated samples. Shown are three independent experiments with error bars denoting SEM. All statistical significances were calculated using one-tailed Student's t-tests, $* \mathrm{p} \leq 0.05$, ** $\leq$ 0.01 , and $* * * \mathrm{p} \leq 0.001$.

Fig. 6. Downregulation of mt-dsRNAs reduces the immune response to poly I:C stimulation. (A) Heatmap of mRNA sequencing results for type I IFN signaling pathway genes. The two columns represent $\log _{2}$ fold changes with or without 2 -CM pretreatment $24 \mathrm{~h}$ prior to poly I:C 
stimulation. (B) APH assay showing cell viability upon poly I:C stimulation with or without 2CM pretreatment. Shown are four independent experiments with error bars denoting SEM. (C) Western blot analysis of PKR phosphorylation upon poly I:C stimulation with or without 2-CM pretreatment. (D) Representative fluorescence images of ZO-1 and Occludin upon poly I:C stimulation with or without 2-CM pretreatment. AQP5-GFP expression upon poly I:C stimulation with or without 2-CM pretreatment was analyzed by (E) western blot and (F) immunocytochemistry. The fluorescence intensities in $(\mathbf{D})$ and $(\mathbf{F})$ were quantified as the mean of three independent experiments and normalized to the Mock transfection control group. The scale bar represents $100 \mu \mathrm{m}$. All statistical significances were calculated using one-tailed Student's ttests, $* \mathrm{p} \leq 0.05, * * \mathrm{p} \leq 0.01$, and $* * * \mathrm{p} \leq 0.001$

\section{Graphical Abstract. mt-dsRNAs as the molecular mediator of autoimmune phenotypes in}

SS. mt-dsRNAs are elevated in both human samples and mouse model of SS and function to exacerbate dsRNA-induced autoimmune phenotypes in SS. Countering the accumulation of mtdsRNAs alleviates the autoimmune phenotypes in SGECs. 
Figure 2.

A

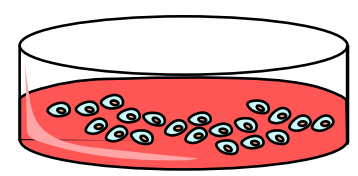

NS-SV-AC cells growing in 2D

B

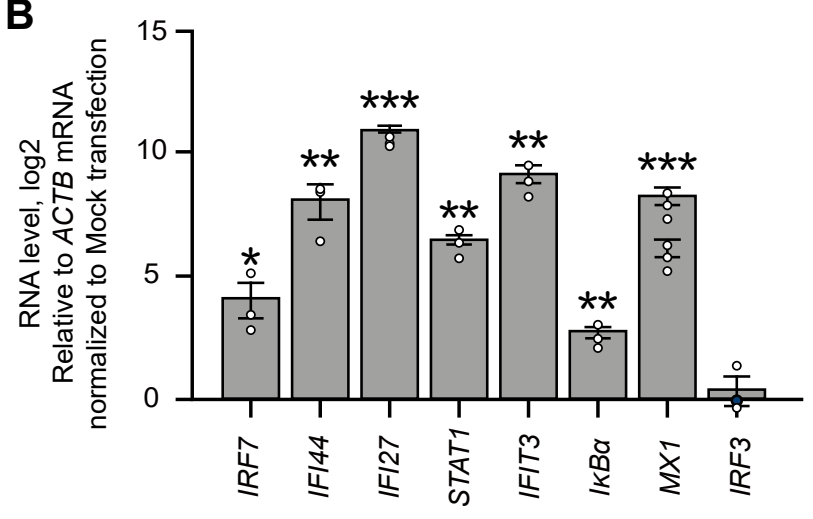

Clear expression of tight junction complex proteins and aquaporin-5 in pV4D4-induced spheroids

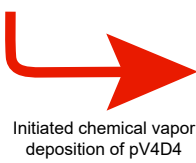

(2) $\left.\begin{array}{c}\text { Tight junction complex } \\ \text { proteins (Occludin, ZO-1) }\end{array}\right\}\{$ Aquaporin 5 (AQP5)

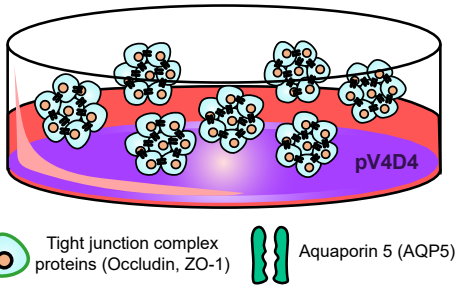

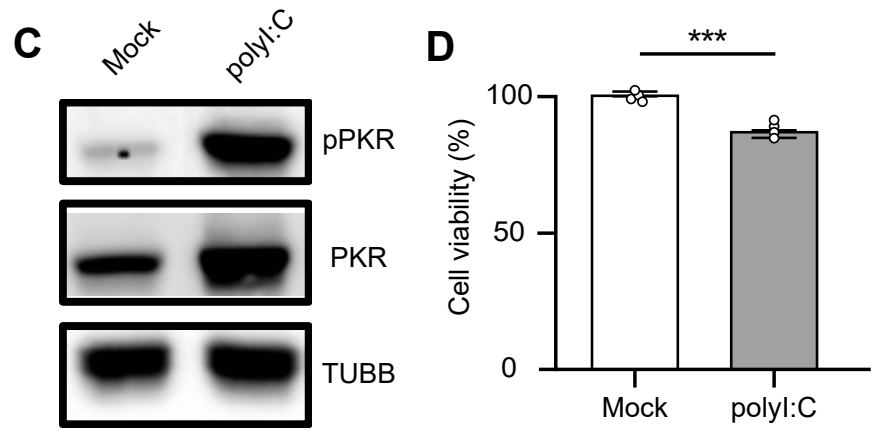

$E$
을
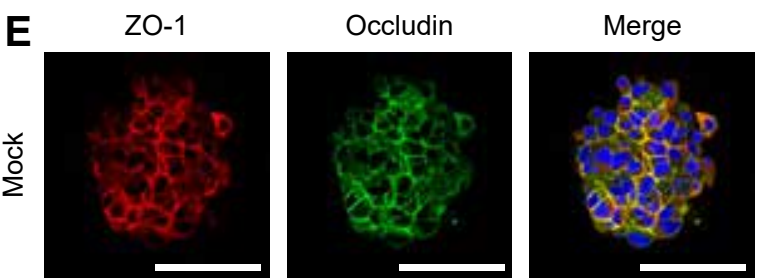

पํํำ
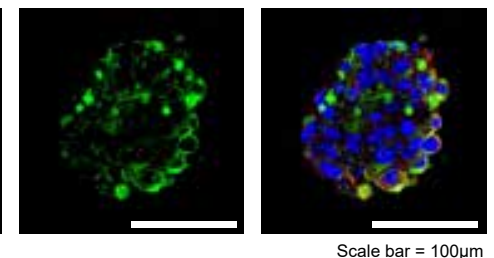

F

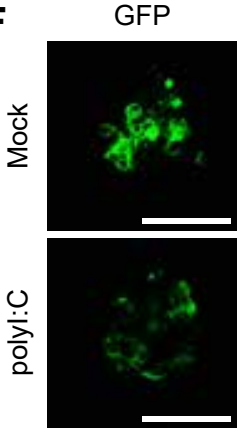

Merge
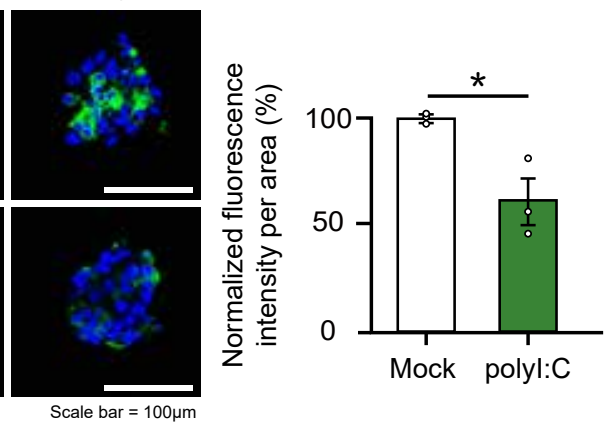

G

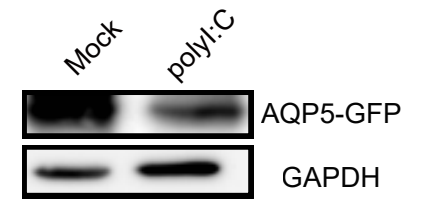

H

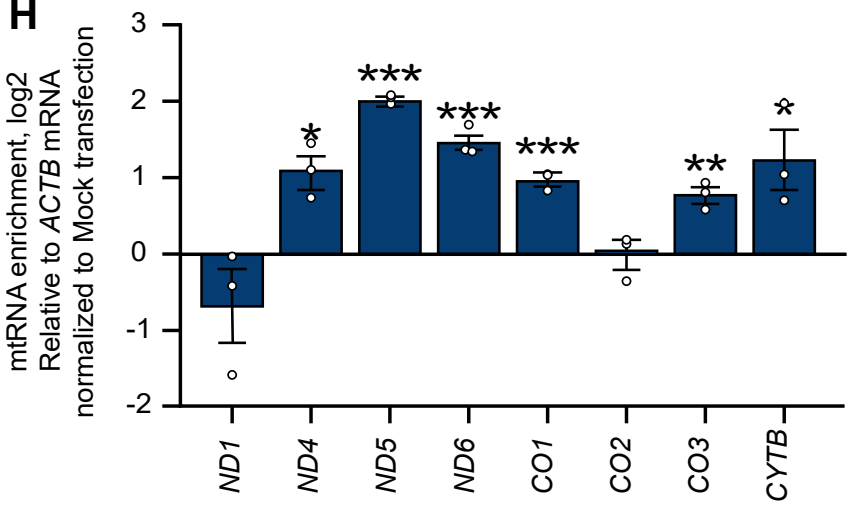


Figure 3.

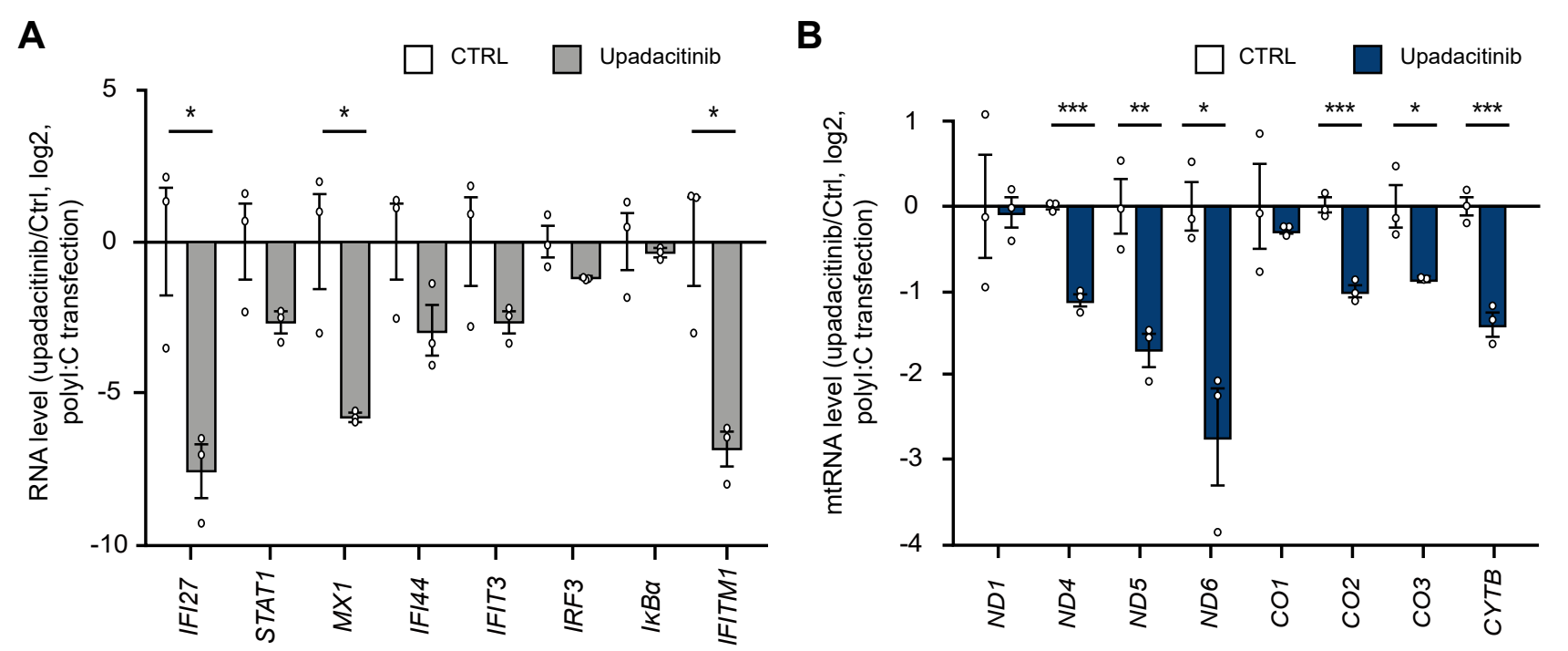


Figure 4.

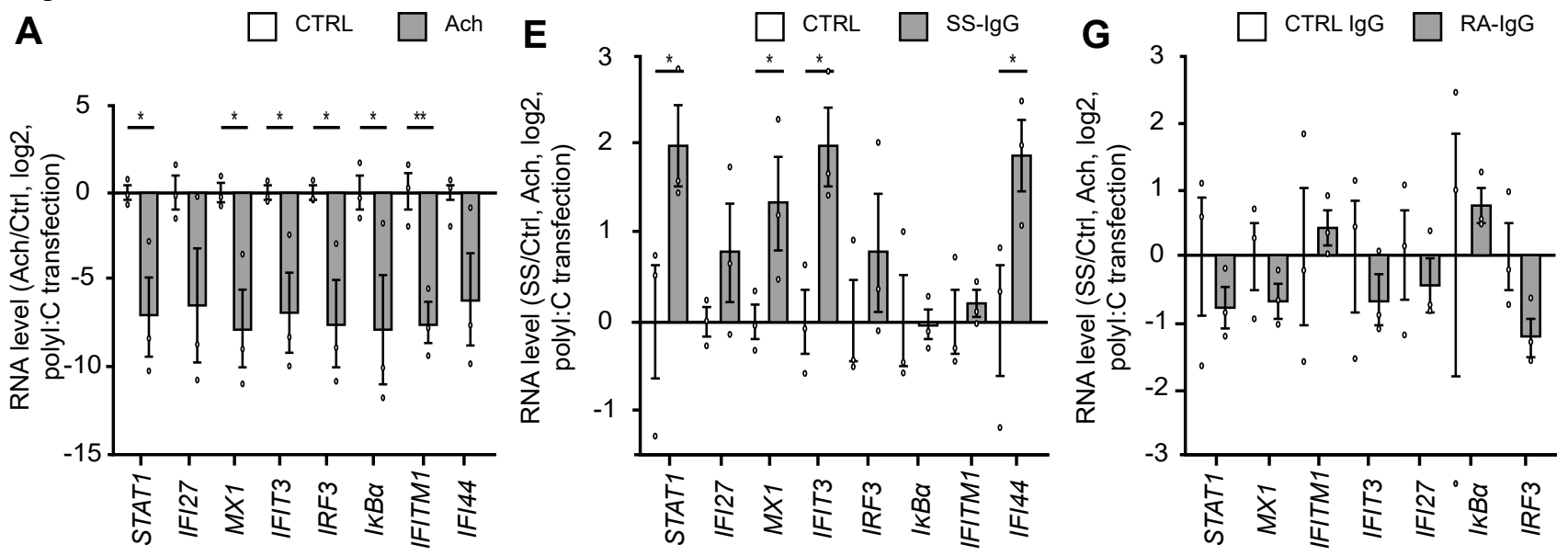
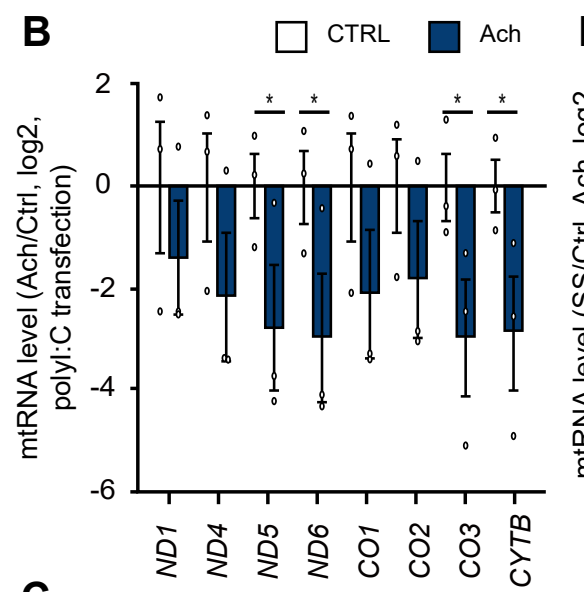

C

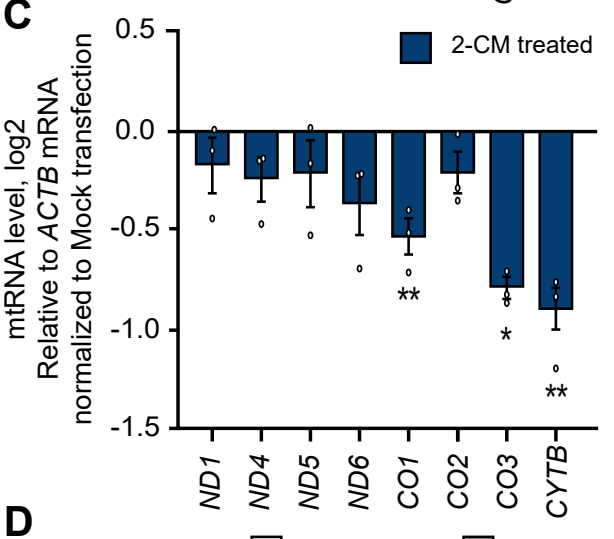

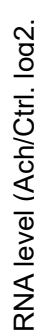

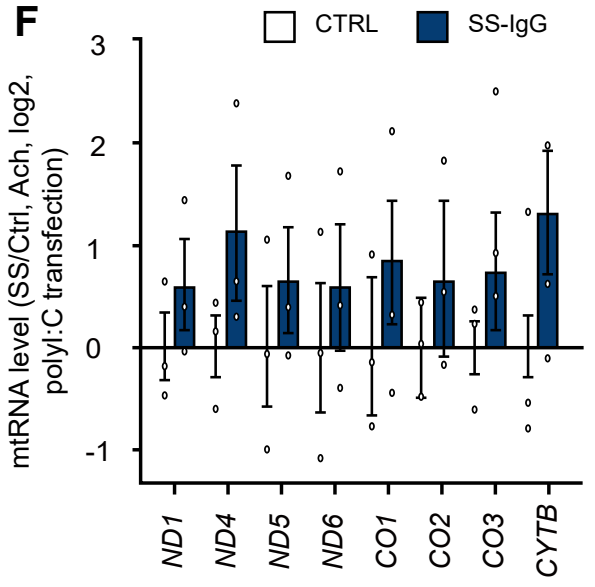

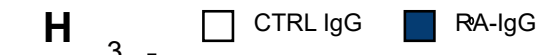

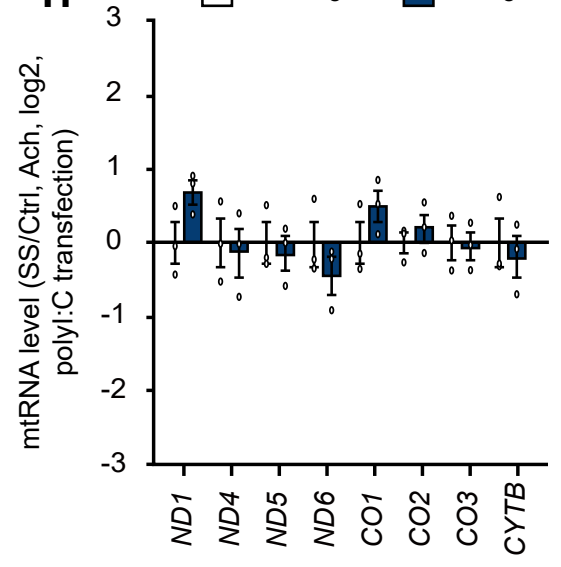

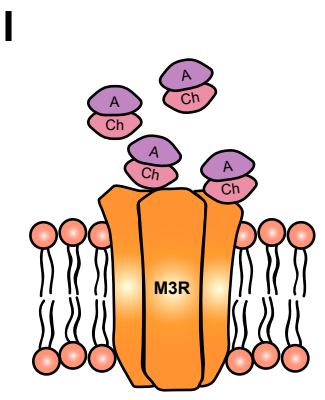

Healthy control
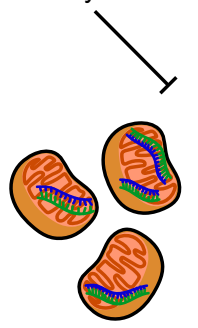

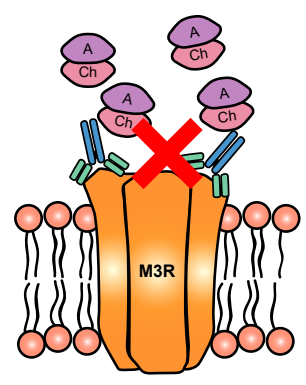

SS patients

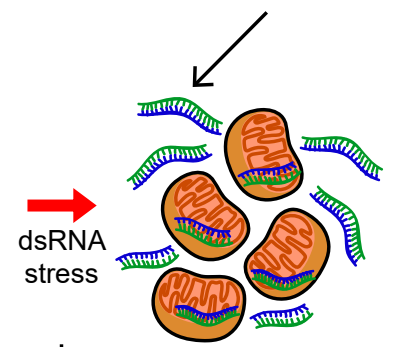

$\downarrow$ mt-dsRNA enrichment

Immune activation triggered by mt-dsRNA 


\section{Figure 5.}

A

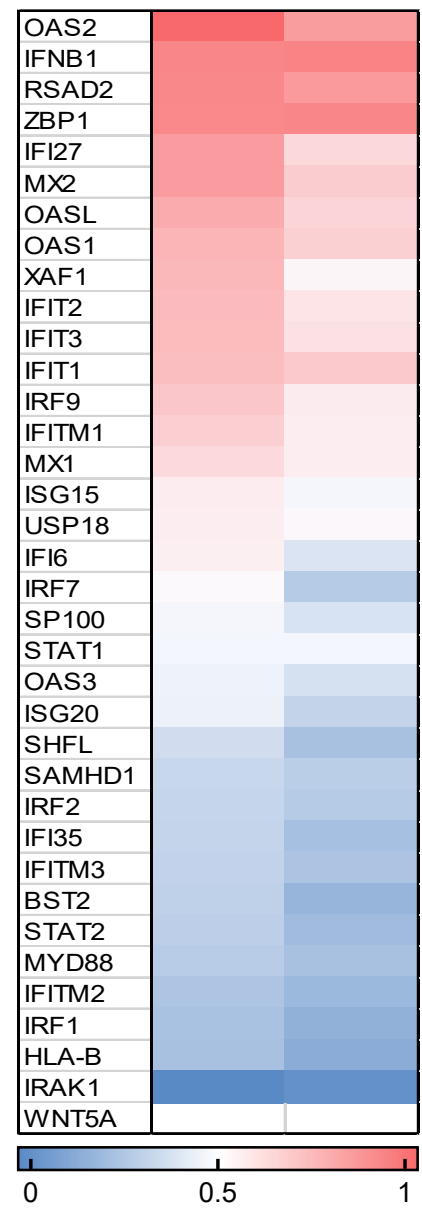

Normalized log2 fold changes

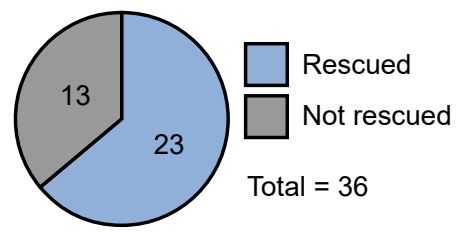

B

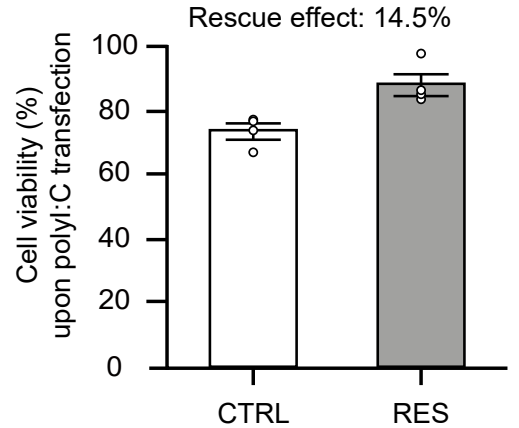

C
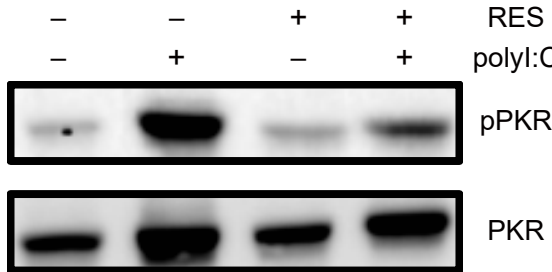

PKR

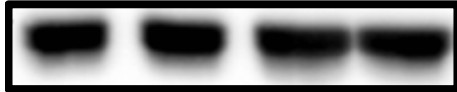

TUBB

D DMSO
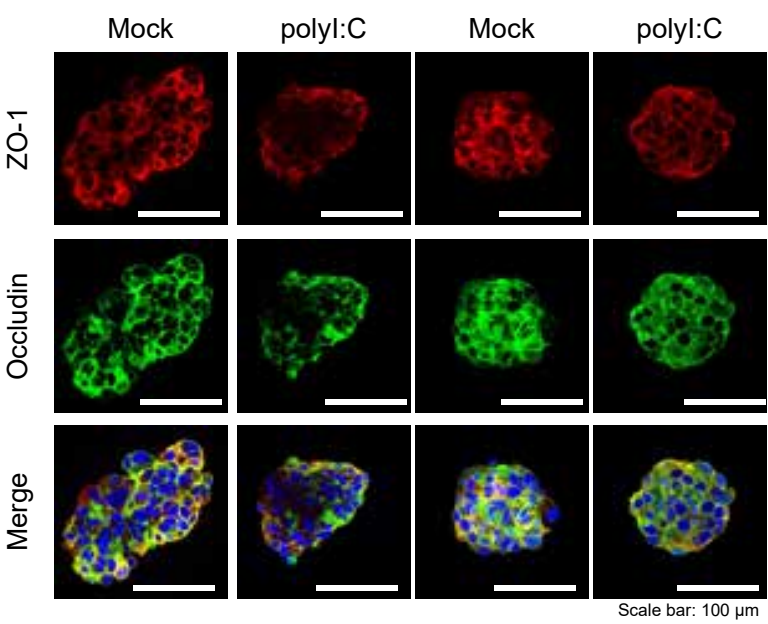

Normalized fluorescence intensity per area (\%) upon polyl:C transfection

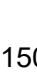

ZO-1

Occludin
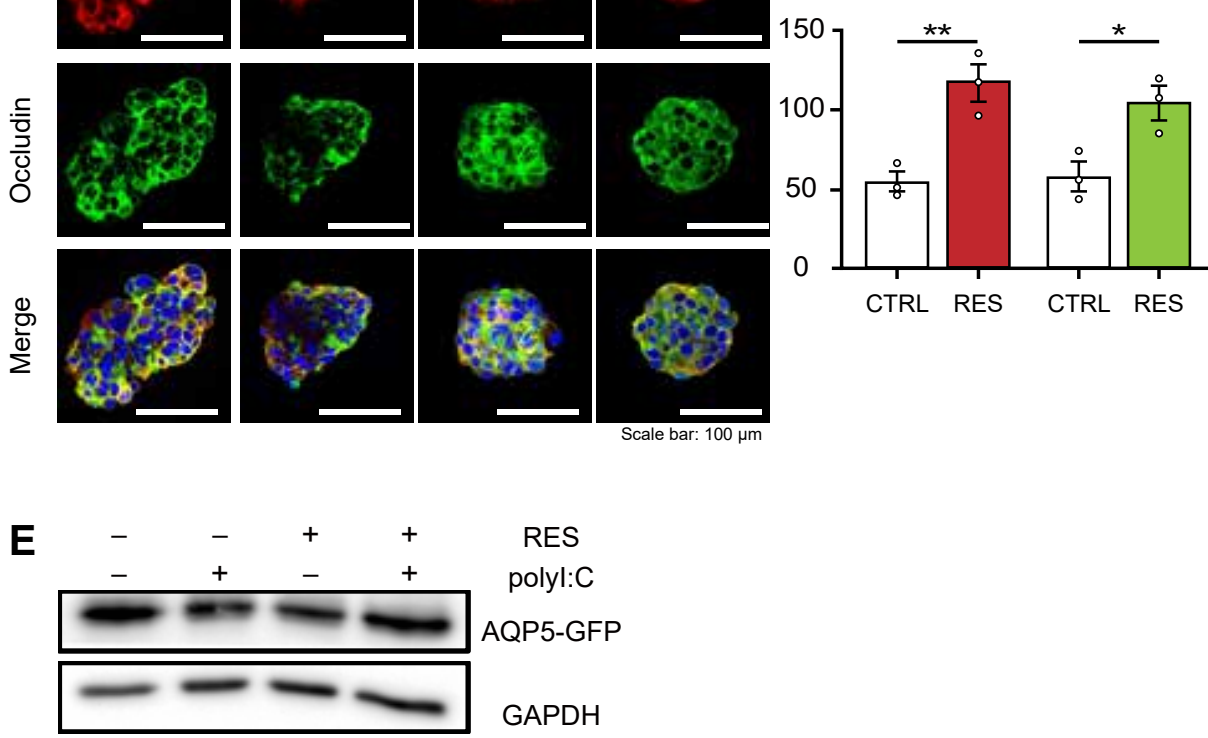

F

$$
\begin{aligned}
& 0 \\
& \frac{1}{1} \\
& 0 \\
& 0 \\
& 0 \\
& 0
\end{aligned}
$$

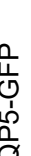

DMSO

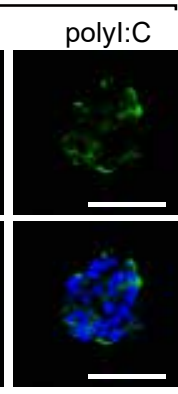

RES

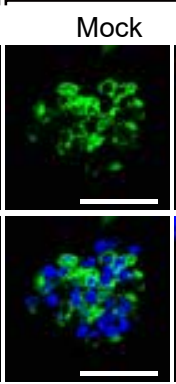

Normalized fluorescence intensity per area (\%) upon

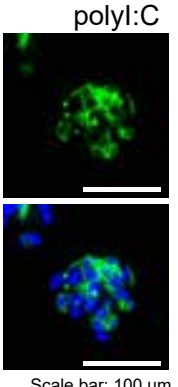
polyl:C transfection

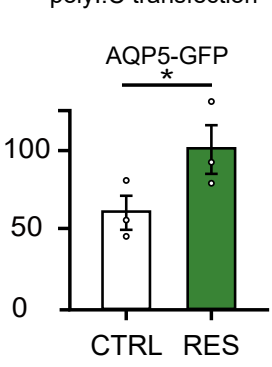

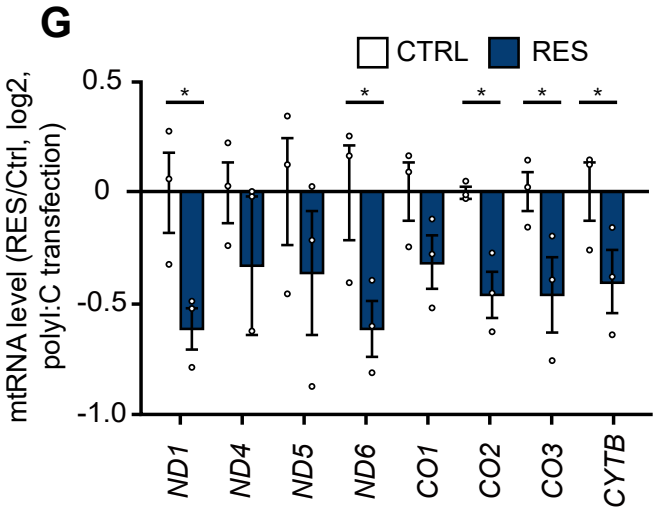




\section{Graphical Abstract}

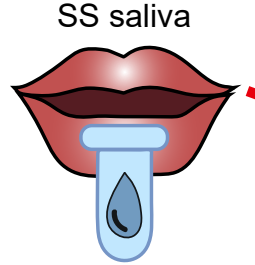

SS tear

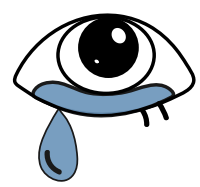

NOD mice salivary gland

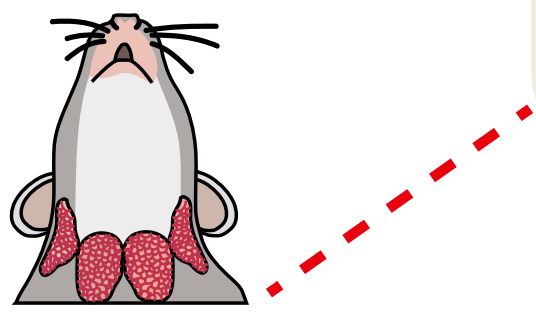

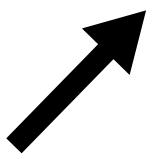

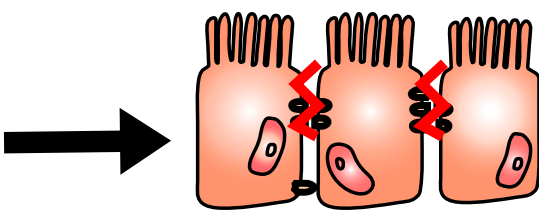

Disruption in tight junction complex

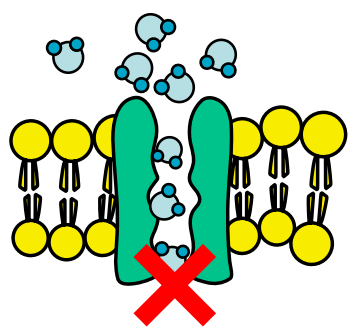

Decrease in AQP5 water-channel protein 